\title{
A time-stepping method for stiff multibody dynamics with contact and friction
}

\author{
Mihai Anitescu ${ }^{1, * \dagger}$ and Florian A. Potra ${ }^{2}$ \\ ${ }^{1}$ Department of Mathematics, University of Pittsburgh, Thackeray 301, Pittsburgh, PA 15213, U.S.A. \\ ${ }^{2}$ Department of Mathematics, University of Maryland, Baltimore County, Baltimore, MD 21250, U.S.A.
}

\begin{abstract}
SUMMARY
We define a time-stepping procedure to integrate the equations of motion of stiff multibody dynamics with contact and friction. The friction and non-interpenetration constraints are modelled by complementarity equations. Stiffness is accommodated by a technique motivated by a linearly implicit Euler method. We show that the main subproblem, a linear complementarity problem, is consistent for a sufficiently small time step $h$. In addition, we prove that for the most common type of stiff forces encountered in rigid body dynamics, where a damping or elastic force is applied between two points of the system, the method is well defined for any time step $h$. We show that the method is stable in the stiff limit, unconditionally with respect to the damping parameters, near the equilibrium points of the springs. The integration step approaches, in the stiff limit, the integration step for a system where the stiff forces have been replaced by corresponding joint constraints. Simulations for one- and two-dimensional examples demonstrate the stable behaviour of the method. Published in 2002 by John Wiley \& Sons, Ltd.
\end{abstract}

KEY WORDS: multibody dynamics; rigid bodies; Coulomb friction; stiff methods; linear complementarity problems; linearly implicitly integration

\section{INTRODUCTION}

The dynamic rigid multibody contact problem is concerned with predicting the motion of several rigid bodies in contact. Work in a number of research areas, robotics and virtual reality especially, has recently led to a strong interest in this problem. Friction is a fundamental phenomenon exhibited at the contact between two bodies, and its accurate modelling is important in various applications, such as a robot grasping a load [1]. Possibly, the most accepted model of dry friction is the Coulomb friction model. The major obstacle in

*Correspondence to: Mihai Anitescu, Department of Mathematics, University of Pittsburgh, Thackeray 301, Pittsburgh, PA 15260, U.S.A.

†E-mail: anitescu@math.pitt.edu

$¥$ This article is a U.S. Government work and is in the public domain in the U.S.A.

Contract/grant sponsor: U.S. Department of Energy; contract/grant number: W-31-109-Eng-38

Contract/grant sponsor: National Science Foundation; contract/grant number: DMS-9973071

Contract/grant sponsor: National Science Foundation; contract/grant number: DMS-9996154

Published in 2002 by John Wiley \& Sons, Ltd.

Received 24 May 2001

Revised 12 September 2001 
incorporating the Coulomb friction model in multibody dynamics simulation is that the classical force-acceleration model with a corresponding Newton law is inconsistent: it does not necessarily have a solution in the classical sense $[2,3]$.

Several approaches have been designed to circumvent this inconsistency while simulating the dynamics of several bodies with intermittent contact and stick-slip motion due to friction.

- The simulation can be interpreted as a succession of differential algebraic equations (DAEs), and certain event functions (such as the distance between two bodies) are used to decide when the DAE needs to be changed [4]. Unfortunately, there is no guarantee that the new DAE formulation will satisfy the frictional and geometric constraints.

- The acceleration equations, which constitute a linear complementarity problem, can be solved by Lemke's algorithm [5]. The outcome of Lemke's algorithm can be translated either in a solution of the acceleration equation or in an unbounded ray that can be transformed in a kinematically but not necessarily dynamically feasible trajectory [2].

- The friction force can be estimated from previous history by using some quadrature or extrapolation rule, and one can solve for the remaining unknowns from the acceleration equations. The reduced problem is a convex problem that can be solved fairly efficiently [6]. The resolution of the model under this approach is not always dynamically correct, but it is usually acceptable.

Recently, an alternative approach has been proposed. Recognizing that the nature of the frictional constraint can induce discontinuous, impulsional behaviour of the bodies involved in the contact configuration, the new approach considers impulses and velocities as the fundamental unknowns $[7,8]$. This framework is based on a linear complementarity problem (LCP), but it is different from previous approaches that attempt to find the accelerations of the bodies $[9,10]$. Previous approaches solve for accelerations from the dynamics equations and then use the accelerations in an integration procedure. In the new framework, the integration and dynamical resolution steps are combined. The main achievement of this approach is that it has solutions for any configuration [7]. As the time-step tends to zero, a subsequence of the numerical solutions approaches the solution of a differential inclusion [3].

The approach of References [7,8] uses Euler's method as the fundamental integration procedure. This is a major obstacle when handling stiff systems with contact and friction. Such systems are interesting in the context of stabilized fixtures, for example, Reference [11]. It is therefore important to modify this scheme to accommodate stiffness in a manner that preserves its well-posedness.

\subsection{The original semi-implicit time-stepping scheme for non-stiff systems}

We first show that the impulse-velocity time-stepping scheme [7,8] can be interpreted as a semi-implicit Euler method applied to the appropriate differential complementarity problem (DCP). This will justify our treatment of stiffness as a natural extension of the similar stiff DAE approach.

We first describe the acceleration-force contact and friction model. An important part will be played by complementarity constraints. Two vectors $a$ and $b$ are called complementary if they satisfy

$$
a \geqslant 0, \quad b \geqslant 0 \quad \text { and } \quad a^{\mathrm{T}} b=0
$$


where all the inequalities are understood componentwise. Alternatively, we may denote a complementarity relation by

$$
a \geqslant 0 \perp b \geqslant 0
$$

We assume that the state of the system of rigid bodies can be described by a generalized position vector $q$ and a generalized velocity vector $v$. The system is subject to several constraints.

Equality constraints. The dynamics of the system must satisfy certain equality constraints, such as those generated by a revolute joint between two bodies [12]. Such constraints are described by

$$
\Theta^{(i)}(q)=0, \quad i=1,2, \ldots, m
$$

Here, $\Theta^{(i)}(q)$ are sufficiently smooth functions. We denote by $v^{(i)}(q)$ the gradient of the corresponding function, or

$$
v^{(i)}(q)=\nabla_{q} \Theta^{(i)}(q), \quad i=1,2, \ldots, m
$$

The force exerted by a joint on the system is $c_{v}^{(i)} v^{(i)}(q)$, where $c_{v}^{(i)}$ is the appropriate Lagrange multiplier [12].

Non-interpenetration constraints. Two bodies cannot penetrate each other. We assume that we can define a continuous signed distance function between the two bodies $\Phi(q)$. Such a distance function classifies the relative positions in the following manner:

- If $\Phi(q)>0$, then the bodies are separated.

- If $\Phi(q)=0$, then the bodies are in contact.

- If $\Phi(q)<0$, then the bodies interpenetrate each other.

In general, a continuous signed distance function $\Phi(q)$ cannot be determined for all possible configurations of two bodies [13]. However, under certain weak assumptions about the shape of the bodies, such a function can be defined at least in a neighbourhood of all contact configurations [13], which will be sufficient for our developments. If such a function $\Phi$ can be defined for every pair of bodies, then the non-interpenetration constraints become

$$
\Phi^{(j)}(q) \geqslant 0, \quad j=1,2, \ldots, p
$$

During the dynamical evolution of the system, few bodies may actually get to be in contact, so that $p$ may be substantially smaller than the number of all possible choices of pairs of bodies.

The function $\Phi(q)$ is generally not differentiable, especially when the bodies have flat surfaces. This situation is generally remediable by considering different geometric primitives [14]. For example, requiring that the distance between a rectangular body in two dimensions and a flat tabletop be non-negative is equivalent to requiring that the signed distance between every vertex of the rectangle and the tabletop be non-negative. It follows that the signed distance between a point and the tabletop is differentiable everywhere, whereas the distance between the body and the tabletop is not.

Since a general analysis of the modelling of the geometrical configurations and the representation of the non-interpenetration constraints by differentiable functions is beyond the scope 
of this work, we will simply assume that we can represent the non-interpenetration constraints between all bodies in the system by (2), for suitably chosen continuously differentiable functions $\Phi^{(j)}(q)$. For more details about the algebraic representation of non-interpenetration constraints, see Reference [14]. In the sequel, the function $\Phi^{(j)}$ will be called contact $(j)$.

An important object is the normal at a contact constraint,

$$
n^{(j)}(q)=\nabla_{q} \Phi^{(j)}(q), \quad j=1,2, \ldots, p
$$

which is now defined, since we assume the functions to be differentiable.

Contact constraints. If $\Phi^{(j)}(q)=0$ for some index $j$, then a corresponding pair of bodies is in exact contact. In this case, a 'normal' force $c_{n}^{(j)} n^{(j)}(q)$ will act at the contact. The force can be only a compression force, which means that $c_{n}^{(j)} \geqslant 0$. The fact that the force acts only when contact is present can be expressed by the complementarity constraint $c_{n}^{(j)} \Phi^{(j)}(q)=0$. The contact constraints thus become

$$
\Phi^{(j)}(q) \geqslant 0, \quad c_{n}^{(j)} \geqslant 0, \quad \Phi^{(j)}(q) c_{n}^{(j)}=0, \quad j=1,2, \ldots, p
$$

Frictional constraints. For the frictional constraints treatment we adopt the description from Reference [3]. The frictional constraints connect the tangential force, the normal force, and the velocity at some contact $(j)$. In the following discussion we omit the superscript $(j)$, although all the quantities refer to the $(j)$ th contact.

We represent the set of possible friction forces through

$$
\mathrm{FC}_{0}(q)=\left\{\bar{D}(q) \bar{\beta} \mid \bar{\beta} \in \mathbb{R}^{d}, \psi(\bar{\beta}) \leqslant \mu\right\}
$$

The function $\psi(\bar{\beta})$ must be convex, positively homogeneous, and coercive. Here $\mu$ is the friction coefficient, a non-negative quantity. This makes the total force at the contact to be inside the friction cone:

$$
\mathrm{FC}(q)=c_{n}\left(n(q)+\mathrm{FC}_{0}(q)\right)=\left\{c_{n} n(q)+\bar{D}(q) \bar{\beta} \mid \bar{\beta} \in \mathbb{R}^{d}, \psi(\bar{\beta}) \leqslant \mu c_{n}\right\}
$$

If $\bar{D}(q)$ spans the friction plane and $\psi(\bar{\beta})=\|\beta\|_{2}$, then $\mathrm{FC}(q)$ becomes the classical circular friction cone [1]. The current representation, however, also covers the representation in global co-ordinates, where $n(q)$ is not necessarily orthogonal to $\bar{D}(q)$ [13].

The maximal dissipation principle requires that we choose $\bar{\beta}$ so as to maximize the dissipation rate $-v^{\mathrm{T}} \bar{D}(q) \bar{\beta}$ over $\bar{D}(q) \bar{\beta} \in c_{n} \mathrm{FC}_{0}(q)$. That is, $\bar{\beta}$ is the solution of the following optimization problem:

$$
\min _{\bar{\beta} \in \mathbb{R}^{d}} v^{\mathrm{T}} \bar{D}(q) \bar{\beta} \quad \text { subject to } \quad \psi(\bar{\beta}) \leqslant \mu c_{n}
$$

However, for computational purposes, we work with a polyhedral approximation of the friction cone $[3,7,8]$. This approximation is generated by $\left\{n(q)+d_{i}(q), i=1,2, \ldots, m_{\mathrm{C}}\right\}$, where $d_{i}(q)$ is a collection of direction vectors in $\mathrm{FC}_{0}(q)$. We write $D(q)=\left[d_{1}(q), d_{2}(q), \ldots, d_{m_{\mathrm{C}}}(q)\right]$. The set of columns of $D(q)$ is generally chosen to be balanced; that is for any $i$ there is a $j$ such that $d_{j}(q)=-d_{i}(q)$ [8]. In this setup, one non-negative component $\beta_{i}$ is associated with every column $d_{i}(q)$ of $D(q), i=1,2, \ldots, m_{\mathrm{C}}$. We denote the vector of tangential forces by $\beta=\left(\beta_{1}, \beta_{2}, \ldots, \beta_{m_{\mathrm{C}}}\right)^{\mathrm{T}}$. The total tangential force thus becomes $D(q) \beta[7,8]$. 
In terms of these variables, the frictional constraints, including the maximum dissipation principle (5), can be expressed in terms of the following complementarity conditions $[3,7,8]$ :

$$
\begin{array}{r}
D(q)^{\mathrm{T}} v+\lambda e \geqslant 0 \perp \beta \geqslant 0 \\
\mu c_{n}-e^{\mathrm{T}} \beta \geqslant 0 \perp \lambda \geqslant 0
\end{array}
$$

Here $e$ is a vector of ones of dimension $m_{\mathrm{C}}, e=(1,1, \ldots, 1)^{\mathrm{T}}$. The additional variable $\lambda$ is approximately equal to the norm of the tangential velocity at the contact, if there is relative motion at the contact, or $\left\|D(q)^{\mathrm{T}} v\right\| \neq 0[7,8]$.

The acceleration-force framework. If we combine the Newton equation of dynamics with the joint, contact, and frictional constraints, we obtain the following DCP:

$$
\begin{gathered}
M(q) \frac{\mathrm{d}^{2} q}{\mathrm{~d} t^{2}}-\sum_{i=1}^{m} v^{(i)} c_{v}^{(i)}-\sum_{j=1}^{p}\left(n^{(j)}(q) c_{n}^{(j)}+D^{(j)}(q) \beta^{(j)}\right)=k\left(t, q, \frac{\mathrm{d} q}{\mathrm{~d} t}\right) \\
\Theta^{(i)}(q)=0, \quad i=1,2, \ldots, m \\
\Phi^{(j)}(q) \geqslant 0, \quad \perp c_{n}^{(j)} \geqslant 0, j=1,2, \ldots, p \\
D^{(j)}(q)^{\mathrm{T}} v+\lambda^{(j)} e^{(j)} \geqslant 0 \quad \perp \beta^{(j)} \geqslant 0 \\
\mu^{(j)} c_{n}^{(j)}-e^{(j)^{\mathrm{T}}} \beta^{(j)} \geqslant 0, \quad \perp \lambda^{(j)} \geqslant 0, j=1,2, \ldots, p
\end{gathered}
$$

Here $M(q)$ is the mass matrix, which we consider to be symmetric and uniformly positive definite. The quantity $k(t, q, \mathrm{~d} q / \mathrm{d} t)$ is the external force.

Unfortunately, a simple two-dimensional example demonstrates that such a set-up is not always well posed $[2,3]$. Since in two dimensions the friction cone coincides with its polyhedral approximation, this problem does not arise from the discretization of the Coulomb cone. It is simply an indication that the Coulomb friction model is inconsistent with the classical equations of rigid body dynamics.

We ignore for the time being the possible inconsistency of the acceleration-force approach, and we investigate a particular numerical integration method applied to the equations of motion (6). We formulate all geometrical constraints at the velocity level. If we differentiate the joint constraint (1) with respect to time, we obtain $\nabla_{q} \Theta^{(i)}(q)^{\mathrm{T}} v=v^{(i)}(q)^{\mathrm{T}} v=0$. Contact constraints cannot be replaced in the same manner by velocity formulations because $\Phi^{(j)}(q) \geqslant 0$ does not necessarily imply that $(\mathrm{d} / \mathrm{d} t) \Phi^{(j)}(q) \geqslant 0$. However, if the contact constraint is active, $\Phi^{(j)}(q)=0$, then we must necessarily have $(\mathrm{d} / \mathrm{d} t) \Phi^{(j)}(q)=n^{(j)}(q)^{\mathrm{T}} v \geqslant 0$.

For practical reasons, it is useful to think about a larger active contact constraint set. During the integration procedure it is possible that while a contact constraint $(j)$ should be theoretically active, the value of $\Phi^{(j)}$ will not be zero because of numerical error. Such a contact will be considered active. Also, some bodies may collide, generating additional active constraints. In this work we will just assume that the active set $\mathscr{A}$ is provided, and we will not discuss the methods for updating the active set unless this has an immediate consequence for the dynamics resolution problem. For the elements $j$ of the active set $\mathscr{A}$ we replace the contact constraints $\Phi^{(j)}(q) \geqslant 0$ by $n^{(j)}(q)^{\mathrm{T}} v \geqslant 0$.

The semi-implicit time-stepping scheme. Finally, we need an integration procedure. From the positions and velocities $q^{(l)}, v^{(l)}$ at time $t$ we need to obtain the positions and velocities 
$q^{(l+1)}, v^{(l+1)}$ at time $t+h$. To do so, we apply the first-order finite-difference approximation $q^{(l+1)}=q^{(l)}+h v^{(l+1)}$, as it is common in semi-implicit schemes [15]. The force is evaluated at $q^{(l)}, v^{(l)}$ which makes the scheme equivalent to the semi-implicit Euler scheme for the case where there are no constraints. With these choices, our method can be written as

$$
\begin{gathered}
M\left(q^{(l)}\right) \frac{v^{(l+1)}-v^{(l)}}{h}-\sum_{i=1}^{m} v^{(i)} c_{v}^{(i)}-\sum_{j \in \mathscr{A}}\left(n^{(j)} c_{n}^{(j)}+D^{(j)} \beta^{(j)}\right)=k\left(t, q^{(l)}, v^{(l)}\right) \\
v^{(i)^{\mathrm{T}}} v^{(l+1)}=0, \quad i=1,2, \ldots, m \\
\rho^{(j)}=n^{(j)^{\mathrm{T}}} v^{(l+1)} \geqslant 0, \quad \perp c_{n}^{(j)} \geqslant 0, \quad j \in \mathscr{A} \\
\sigma^{(j)}=\lambda^{(j)} e^{(j)}+D^{(j) \mathrm{T}} v^{(l+1)} \geqslant 0, \quad \perp \beta^{(j)} \geqslant 0, j \in \mathscr{A} \\
\zeta^{(j)}=\mu^{(j)} c_{n}^{(j)}-e^{(j)^{\mathrm{T}}} \beta^{(j)} \geqslant 0, \quad \perp \lambda^{(j)} \geqslant 0, \quad j \in \mathscr{A}
\end{gathered}
$$

To simplify notation, we have not explicitly shown dependence on the geometrical data of the problem, $v^{(i)}, n^{(j)}, D^{(j)}$, on $q^{(l)}$. Here and in all other formulations this data is evaluated solely at $q^{(l)}$. The model constitutes a mixed linear complementarity problem (LCP) [5]. We now do the following relabellings: $c_{v}^{(i)} \leftarrow h c_{v}^{(i)}, i=1,2, \ldots, m$, and $c_{n}^{(j)} \leftarrow h c_{n}^{(j)}, \beta^{(j)} \leftarrow h \beta^{(j)}$, and $\zeta^{(j)} \leftarrow h \zeta^{(j)}$ for $j \in \mathscr{A}$.

Then, after multiplying the first equation above by $h$, we finally obtain the following LCP:

$$
\begin{gathered}
M\left(q^{(l)}\right) v^{(l+1)}-\sum_{i=1}^{m} v^{(i)} c_{v}^{(i)}-\sum_{j \in \mathscr{A}}\left(n^{(j)} c_{n}^{(j)}+D^{(j)} \beta^{(j)}\right)=h k\left(t, q^{(l)}, v^{(l)}\right)+M\left(q^{(l)}\right) v^{(l)} \\
v^{(i)^{\mathrm{T}}} v^{(l+1)}=0, \quad i=1,2, \ldots, m \\
\rho^{(j)}=n^{(j)^{\mathrm{T}}} v^{(l+1)} \geqslant 0, \quad \perp c_{n}^{(j)} \geqslant 0, \quad j \in \mathscr{A} \\
\sigma^{(j)}=\lambda^{(j)} e^{(j)}+D^{(j) \mathrm{T}} v^{(l+1)} \geqslant 0, \quad \perp \beta^{(j)} \geqslant 0, j \in \mathscr{A} \\
\zeta^{(j)}=\mu^{(j)} c_{n}^{(j)}-e^{(j)^{\mathrm{T}}} \beta^{(j)} \geqslant 0, \quad \perp \lambda^{(j)} \geqslant 0, \quad j \in \mathscr{A}
\end{gathered}
$$

Note that the last three groups of equations of (7) have remained formally unchanged from the acceleration-force formulation, because of their homogeneity.

We can now interpret the first equation as a relationship between velocities and impulses. The major advantage of this new set-up is that the LCP has now a solution $v^{(l+1)}$ for any choices of the relevant parameters as soon as $M\left(q^{(l)}\right)$ is positive definite, although the original continuous model does not necessarily have a solution [7]. Lemke's algorithm [5] will determine a solution of this LCP in a finite number of steps [7]. If we denote the sequence of positions and velocities produced by the scheme by $q^{(l), h}$ and $v^{(l), h}$, then we can show that, as $h \rightarrow 0$, the respective sequences admit subsequences that satisfy the constraints and a measure differential inclusion formulation of the dynamics equations [3]. Such a relaxation of the model is necessary because, as argued before, the classical model will not always have a solution.

The time-stepping scheme based on (7) will thus be consistent for all configurations. Since the method is essentially equivalent to the semi-implicit Euler method when there are no constraints, it is likely that the method will perform poorly when stiff forces are acting on 
the system. A natural modification, the use of an implicit Euler method, has the disadvantage of generating a nonlinear complementarity problem, which may be considerably harder to solve. An important question that we address in the remainder of the paper is whether we can accommodate stiffness while still solving linear complementarity problems.

\section{A NEW LINEARLY IMPLICIT TIME-STEPPING SCHEME FOR STIFF SYSTEMS}

In this section we introduce a linearly implicit time-stepping scheme for stiff systems with contact and friction constraints. To justify our approach we will first discuss the unconstrained case.

\subsection{The linearly implicit approach for ordinary differential equations}

Take a generic differential equation

$$
\frac{\mathrm{d} y}{\mathrm{~d} t}=f(t, y)
$$

where $y \in \mathbb{R}^{n}, f: \mathbb{R} \times \mathbb{R}^{n} \rightarrow \mathbb{R}^{n}$. For this differential equation consider the linearly implicit Euler method [13]

$$
(I-h J)\left(y^{l+1}-y^{l}\right)=h f\left(t^{l}, y^{l}\right)
$$

where $J=\nabla_{y} f\left(t^{l}, y^{l}\right)$. This is an $A$-stable method whose major computational cost per iteration consists in solving a linear system with matrix $I-h J$.

Now consider the case of the dynamics equations in the absence of constraints:

$$
\frac{\mathrm{d} q}{\mathrm{~d} t}=v, \quad M(q) \frac{\mathrm{d} v}{\mathrm{~d} t}=k(t, q, v)
$$

To apply the linearly implicit scheme (8)-(9), we would have to multiply the second equation through with $M^{-1}(q)$ and compute the derivatives of $M^{-1}(q)$ in setting up the Jacobian of the nonlinear system that has to be solved at each integration step. However, since in most multibody systems the stiffness is mainly due to $k(q, v)$, we will consider the matrix $M(q)$ to be constant when setting up that Jacobian. Then, the linearly implicit scheme becomes

$$
\begin{aligned}
q^{(l+1)}= & q^{(l)}+h v^{(l+1)} \\
M\left(q^{(l)}\right) \frac{v^{(l+1)}-v^{(l)}}{h}= & k\left(q^{(l)}, v^{(l)}\right)+h \nabla_{q} k\left(t^{(l)}, q^{(l)}, v^{(l)}\right) v^{(l+1)} \\
& +\nabla_{v} k\left(t^{(l)}, q^{(l)}, v^{(l)}\right)\left(v^{(l+1)}-v^{(l)}\right)
\end{aligned}
$$

or, after solving for $v^{(l+1)}$,

$$
\begin{aligned}
q^{(l+1)}= & q^{(l)}+h v^{(l+1)} \\
v^{(l+1)}= & {\left[M\left(q^{(l)}\right)-h^{2} \nabla_{q} k\left(t^{(l)}, q^{(l)}, v^{(l)}\right)-h \nabla_{v} k\left(t^{(l)}, q^{(l)}, v^{(l)}\right)\right]^{-1} } \\
& \times\left[M\left(q^{(l)}\right) v^{(l)}+h k\left(t^{(l)}, q^{(l)}, v^{(l)}\right)-h \nabla_{v} k\left(t^{(l)}, q^{(l)}, v^{(l)}\right) v^{(l)}\right]
\end{aligned}
$$


By introducing the notations

$$
\begin{aligned}
\widehat{M^{(l)}} & =\left[M\left(q^{(l)}\right)-h^{2} \nabla_{q} k\left(t^{(l)}, q^{(l)}, v^{(l)}\right)-h \nabla_{v} k\left(t^{(l)}, q^{(l)}, v^{(l)}\right)\right] \\
\widehat{k^{(l)}} & =k\left(t^{(l)}, q^{(l)}, v^{(l)}\right)-\nabla_{v} k\left(t^{(l)}, q^{(l)}, v^{(l)}\right) v^{(l)}
\end{aligned}
$$

the linearly implicit Euler step becomes

$$
\begin{aligned}
q^{(l+1)} & =q^{(l)}+h v^{(l+1)} \\
\widehat{M^{(l)}} v^{(l+1)} & =M\left(q^{(l)}\right) v^{(l)}+h \widehat{k^{(l)}}
\end{aligned}
$$

These concepts can naturally be included in the constrained framework. From the Euler LCP step (7) it follows that the natural substitution is $M\left(q^{(l)}\right) v^{(l+1)} \leftarrow M\left(q^{(l)}\right) v^{l+1}$ and $k\left(t^{(l)}, q^{(l)}, v^{(l)}\right) \leftarrow \widehat{k^{(l)}}$. The linearly implicit Euler formulation then becomes $q^{(l+1)}=q^{(l)}+$ $h v^{(l+1)}$, where $v^{(l+1)}$ is the solution of the following mixed LCP

$$
\begin{gathered}
\widehat{M^{(l)}} v^{(l+1)}-\sum_{i=1}^{m} v^{(i)} c_{v}^{(i)}-\sum_{j \in \mathscr{A}}\left(n^{(j)} c_{n}^{(j)}+D^{(j)} \beta^{(j)}\right)=h \widehat{k^{(l)}}+M\left(q^{(l)}\right) v^{(l)} \\
v^{(i)^{\mathrm{T}}} v^{(l+1)}=0, \quad i=1,2, \ldots, m \\
\rho^{(j)}=n^{(j)^{\mathrm{T}}} v^{(l+1)} \geqslant 0, \quad \perp c_{n}^{(j)} \geqslant 0, j \in \mathscr{A} \\
\sigma^{(j)}=\lambda^{(j)} e^{(j)}+D^{(j) \mathrm{T}} v^{(l+1)} \geqslant 0, \quad \perp \beta^{(j)} \geqslant 0, \quad j \in \mathscr{A} \\
\zeta^{(j)}=\mu^{(j)} c_{n}^{(j)}-e^{(j)^{\mathrm{T}}} \beta^{(j)} \geqslant 0, \quad \perp \lambda^{(j)} \geqslant 0, \quad j \in \mathscr{A}
\end{gathered}
$$

In matrix form, this LCP can be written as

$$
\begin{gathered}
{\left[\begin{array}{ccccc}
\widehat{M^{(l)}} & -\tilde{v} & -\tilde{n} & -\tilde{D} & 0 \\
\tilde{v}^{\mathrm{T}} & 0 & 0 & 0 & 0 \\
\tilde{n}^{\mathrm{T}} & 0 & 0 & 0 & 0 \\
\tilde{D}^{\mathrm{T}} & 0 & 0 & 0 & \tilde{E} \\
0 & 0 & \tilde{\mu} & -\tilde{E}^{\mathrm{T}} & 0
\end{array}\right]\left[\begin{array}{c}
v^{(l+1)} \\
\tilde{c}_{v} \\
\tilde{c}_{n} \\
\tilde{\beta} \\
\tilde{\lambda}
\end{array}\right]+\left[\begin{array}{c}
-M v^{(l)}-h \widehat{k^{(l)}} \\
0 \\
0 \\
0 \\
0
\end{array}\right]=\left[\begin{array}{c}
0 \\
0 \\
\tilde{\rho} \\
\tilde{\sigma} \\
\tilde{\zeta}
\end{array}\right]} \\
{\left[\begin{array}{c}
\tilde{c}_{n} \\
\tilde{\beta} \\
\tilde{\lambda}
\end{array}\right]^{\mathrm{T}}\left[\begin{array}{c}
\tilde{\rho} \\
\tilde{\sigma} \\
\tilde{\zeta}
\end{array}\right]=0, \quad\left[\begin{array}{c}
\tilde{c}_{n} \\
\tilde{\beta} \\
\tilde{\lambda}
\end{array}\right] \geqslant 0, \quad\left[\begin{array}{c}
\tilde{\rho} \\
\tilde{\sigma} \\
\tilde{\zeta}
\end{array}\right] \geqslant 0}
\end{gathered}
$$

Here $\tilde{v}=\left[v^{(1)}, v^{(2)}, \ldots, v^{(m)}\right], \quad \tilde{c}_{v}=\left[c_{v}^{(1)}, c_{v}^{(2)}, \ldots, c_{v}^{(m)}\right]^{\mathrm{T}}, \quad \tilde{n}=\left[n^{\left(j_{1}\right)}, n^{\left(j_{1}\right)}, \ldots, n^{\left(j_{s}\right)}\right], \quad \tilde{c}_{n}=\left[c_{n}^{\left(j_{1}\right)}, c_{n}^{\left(j_{2}\right)}\right.$, $\left.\ldots, c_{n}^{\left(j_{s}\right)}\right]^{\mathrm{T}}, \quad \tilde{\beta}=\left[\beta^{\left(j_{1}\right) \mathrm{T}}, \beta^{\left(j_{2}\right) \mathrm{T}}, \ldots, \beta^{\left(j_{s}\right) \mathrm{T}}\right], \quad \tilde{D}=\left[D^{\left(j_{1}\right)}, D^{\left(j_{2}\right)}, \ldots, D^{\left(j_{s}\right)}\right], \quad \tilde{\lambda}=\left[\lambda^{\left(j_{1}\right)}, \lambda^{\left(j_{2}\right)}, \ldots, \lambda^{\left(j_{s}\right)}\right]$, $\tilde{\mu}=\operatorname{diag}\left(\mu^{\left(j_{1}\right)}, \mu^{\left(j_{2}\right)}, \ldots, \mu^{\left(j_{s}\right)}\right)^{\mathrm{T}}$, and $\tilde{D}=\operatorname{diag}\left(e^{\left(j_{1}\right)}, e^{\left(j_{2}\right)}, \ldots, e^{\left(j_{s}\right)}\right)$ are the lumped LCP data, and 
$\mathscr{A}=\left\{j_{1}, j_{2}, \ldots, j_{s}\right\}$ are the active contact constraints. The vector inequalities in (18) are to be understood componentwise.

An important question is whether the LCP (17)-(18) has a solution and, if so, what kind of algorithm can be used to compute it. If the matrix $\widehat{M^{(l)}}(10)$ is symmetric and positive definite, the answer is affirmative [7]. However, for general forces $k(t, q, v)$, symmetry is not expected. Hence, the matrix $\widehat{M^{(l)}}$ is potentially non-symmetric for any value of the time step $h$. The following result ensures that, even for a non-symmetric but positive definite matrix $\widehat{M^{(l)}}$, the LCP (17)-(18) is still solvable by Lemke's algorithm.

\section{Theorem 2.1}

Consider a (mixed) LCP of the form

$$
\begin{gathered}
\left(\begin{array}{l}
0 \\
0 \\
s
\end{array}\right)=\left(\begin{array}{ccc}
M & -F & -H \\
F^{\mathrm{T}} & 0 & 0 \\
H^{\mathrm{T}} & 0 & N
\end{array}\right)\left(\begin{array}{l}
x \\
y \\
\lambda
\end{array}\right)+\left(\begin{array}{c}
-k \\
0 \\
b
\end{array}\right) \\
s \geqslant 0, \quad \lambda \geqslant 0, \quad \lambda^{\mathrm{T}} s=0
\end{gathered}
$$

where $M, N, F, H$ are given matrices and $b, k$ are given vectors of the appropriate dimension.

If $M$ is a positive definite matrix, $N$ a copositive matrix [5, Definition 3.8.1], and $b$ a non-negative vector (in particular, all components of $b$ can be 0 ), then the above LCP has a solution. Lemke's algorithm, with precautions taken against cycling, will always find a solution $(s, \lambda)$ of the LCP obtained by eliminating $x$ and $y$. Then a solution $(x, y, s, \lambda)$ of the original LCP can be recovered by solving for $x$ and $y$ in the first two rows of (19).

Some fundamental notions from the theory of LCP. A matrix $M$ is said to be positive (semi)definite if $x^{\mathrm{T}} M x(\geqslant)>0$ whenever $x \neq 0$, which we also denote by $M(\geqslant) \succ 0$. A matrix $M$ is said to be copositive if $x^{\mathrm{T}} M x \geqslant 0$ whenever $x \geqslant 0$, where the last inequality is understood componentwise. A matrix $M$ with non-negative entries is clearly a copositive matrix. If $M_{1}$ is a positive semi-definite matrix and $M_{2}$ is a copositive matrix, then $M_{1}+M_{2}$ is a copositive matrix. Let $M$ be a copositive matrix, and denote by $\Lambda(M, b)$ the solution set of the linear complementarity problem

$$
s=M x+b \geqslant 0 \quad \perp x \geqslant 0
$$

If for any $z \in \Lambda(M, 0)$, we have that $z^{\mathrm{T}} b \geqslant 0$ then the solution set of the original LCP, $\Lambda(M, b)$ is not empty, and an element of $\Lambda(M, b)$ can be found by Lemke's algorithm [5, Corollary 4.4.12]. This result will be the key of the well-posedness of the time-stepping scheme (12)-(16). Lemke's algorithm is a pivotal algorithm for LCP similar in philosophy to the simplex algorithm of linear programming [5].

\section{Proof of Theorem 2.1.}

We can assume without loss of generality that $F^{\mathrm{T}}$ is full row rank. If not, we can consider just a maximal set of independent rows as being $F^{\mathrm{T}}$. The components of $y$ corresponding to the dependent variables can be set to 0 , and $\lambda$ will be in the same linear space determined 
by (19). After we solve for $x$ in (19), the LCP becomes

$$
\begin{gathered}
0=F^{\mathrm{T}} M^{-1} F y+F^{\mathrm{T}} M^{-1} H \lambda+F^{\mathrm{T}} M^{-1} k \\
s=H^{\mathrm{T}} M^{-1} F y+H^{\mathrm{T}} M^{-1} H \lambda+N \lambda+H^{\mathrm{T}} M^{-1} k+b \\
s \geqslant 0, \quad \lambda \geqslant 0, \quad \lambda^{\mathrm{T}} s=0
\end{gathered}
$$

Since $F$ is full row rank, $F^{\mathrm{T}} M^{-1} F$ is non-singular. Therefore, we can solve for $y$ from the first equation to get the LCP:

$$
\begin{gathered}
s=(G+N) \lambda+g \\
s \geqslant 0, \quad \lambda \geqslant 0, \quad \lambda^{\mathrm{T}} s=0
\end{gathered}
$$

where

$$
\begin{aligned}
& G=H^{\mathrm{T}} M^{-1} H-H^{\mathrm{T}} M^{-1} F\left(F^{\mathrm{T}} M^{-1} F\right)^{-1} F^{\mathrm{T}} M^{-1} H \\
& g=-H^{\mathrm{T}} M^{-1} F\left(F^{\mathrm{T}} M^{-1} F\right)^{-1} F^{\mathrm{T}} M^{-1} k+H^{\mathrm{T}} M^{-1} k+b
\end{aligned}
$$

By construction $G$ is the Schur complement of $N$ in the big matrix of (19), if $N=0$ [5, Definition 2.3.4]. Since the matrix in (19) is positive semi-definite for $N=0$, so is $G$ [5, Theorem 4.1.5]. Therefore, $G+N$ is copositive, since $N$ is copositive.

It is convenient to denote the above $\operatorname{LCP}$ as $\operatorname{LCP}(G+N, g)$ and to call $\lambda$ its solution. Once $\lambda$ is found, then $s=(G+N) \lambda+g$. Let now $z$ be a solution of $\operatorname{LCP}(G+N, 0)$. Then $z^{\mathrm{T}} G z=0$, which also implies that $z^{\mathrm{T}} G^{\mathrm{T}} z=0$. From the expression of $G$ we have

$$
G^{\mathrm{T}}=H^{\mathrm{T}} M^{-\mathrm{T}} H-H^{\mathrm{T}} M^{-\mathrm{T}} F\left(F^{\mathrm{T}} M^{-\mathrm{T}} F\right)^{-1} F^{\mathrm{T}} M^{-\mathrm{T}} H
$$

where we use the traditional notation $M^{-\mathrm{T}}=\left(M^{-1}\right)^{\mathrm{T}}$. Let now $w=-\left(F^{\mathrm{T}} M^{-\mathrm{T}} F\right)^{-1} F^{\mathrm{T}} M^{-\mathrm{T}} H z$, which can also be rewritten as $F^{\mathrm{T}} M^{-\mathrm{T}} F w=-F^{\mathrm{T}} M^{-\mathrm{T}} H z$. From the definition of $w$ and of $G^{\mathrm{T}}$ it follows that $G^{\mathrm{T}} z=H^{\mathrm{T}} M^{-\mathrm{T}} H z+H^{\mathrm{T}} M^{-\mathrm{T}} F w$. Putting these relations in matrix form, we obtain

$$
\left[\begin{array}{c}
G^{\mathrm{T}} z \\
0
\end{array}\right]=\left[\begin{array}{cc}
H^{\mathrm{T}} M^{-\mathrm{T}} H & H^{\mathrm{T}} M^{-\mathrm{T}} F \\
F^{\mathrm{T}} M^{-\mathrm{T}} H & F^{\mathrm{T}} M^{-\mathrm{T}} F
\end{array}\right]\left[\begin{array}{c}
z \\
w
\end{array}\right]=[H F]^{\mathrm{T}} M^{-\mathrm{T}}[H F]\left[\begin{array}{c}
z \\
w
\end{array}\right]
$$

Left-multiplying this relation by $\left[z^{\mathrm{T}}, w^{\mathrm{T}}\right]$, we obtain

$$
0=z^{\mathrm{T}} G^{\mathrm{T}} z=\left([H F]\left[\begin{array}{c}
z \\
w
\end{array}\right]\right)^{\mathrm{T}} M^{-\mathrm{T}}[H F]\left[\begin{array}{c}
z \\
w
\end{array}\right]=(H z+F w)^{\mathrm{T}} M^{-\mathrm{T}}(H z+F w)
$$

Since $M$ is a positive definite matrix, so are $M^{-1}$ and $M^{-\mathrm{T}}$. The last equation therefore implies that $H z+F w=0$. Since $z \geqslant 0$ and $b \geqslant 0$, we deduce that $z^{\mathrm{T}} g=w^{\mathrm{T}} F^{\mathrm{T}} M^{-1} k+z^{\mathrm{T}} H^{\mathrm{T}} M^{-1} k+$ $z^{\mathrm{T}} b=(F w+H z)^{\mathrm{T}} M^{-1} k+z^{\mathrm{T}} b=z^{\mathrm{T}} b \geqslant 0$.

Therefore, we have proved that if $z$ is a solution of $\operatorname{LCP}(G+N, 0)$, then $g^{\mathrm{T}} z \geqslant 0$. Consequently, by Corollary 4.4.12 of Reference [5], Lemke's algorithm, with precautions taken against degeneracy, will find a solution $s, \lambda$ to $\operatorname{LCP}(G+N, g)$ and, by solving for $x$ and $y$ in the first two rows of (19), a solution $(x, y, s, \lambda)$ to the initial LCP. 
Theorem 2.2

For any positive definite mass matrix $M$ and non-negative friction coefficients, the LCP (2.17)-(2.18) has a solution that can be computed by Lemke's algorithm.

Proof

Let $N$ be the matrix made of the last three sets of rows and columns of the matrix of the LCP (17)-(18). Since this matrix is the sum of a positive semi-definite matrix (having $\tilde{E}$ and $-\tilde{E}^{\mathrm{T}}$ as its blocks) and a matrix with non-negative coefficients $(\tilde{\mu})$, it follows that the matrix is copositive. If we define $H=\left[\begin{array}{lll}\tilde{n} & \tilde{D} & 0\end{array}\right]$, the conclusion follows by virtue of Theorem 2.1 .

An important consequence of the Theorem 2.2 is that the time-stepping scheme defined by (12)-(16) will have a solution whenever the matrix $\widehat{M^{(l)}}$ is positive definite.

When friction is involved, the uniqueness of solution cannot be guaranteed by this or any other approach [16].

\subsection{Limits of consistency of the model}

If the mass matrix $M(q)$ is positive definite, then according to $(10), \widehat{M^{(l)}}$ is positive definite for sufficiently small $h$. However, the reason for using a linearly implicit scheme is to obtain as large a time step as possible without encountering instability due to stiffness. We would therefore like to have a guarantee that the method will be allowed to take sufficiently large steps.

It is fairly clear that the method will behave well when there are no constraints, since the linearly implicit Euler method is $A$-stable [15] for classical differential equations. This means, in particular, that the matrix $\widehat{M^{(l)}}$ will have eigenvalues with positive real part for fairly large values of $h$, even for very stiff systems, since its definition does not depend on the existence of the constraints.

The problem, however, is that a matrix whose eigenvalues have positive real part, though non-singular, is not necessarily positive definite, as can be easily seen for the matrix

$$
\left[\begin{array}{cc}
1 & 100 \\
0 & 1
\end{array}\right]
$$

Hence, we cannot necessarily apply Theorem 2.2 in this case for moderate or large values of $h$, although one could reasonably expect its conclusion to hold.

There are, however, two important particular cases in which we can guarantee the positive definiteness of the matrix $\widehat{M^{(l)}}$. In the first case, assume that the external force is of the form $k(q, v)=-\nabla U(q)-\Gamma(v)$, where $\Gamma(v)$ satisfies $\nabla_{v} \Gamma(v) \geqslant 0$ (is a damping-type force) and $U(q)$ is a potential function. If $U(q)$ is a non-degenerate potential function, then we can expect that, near an equilibrium point, $\nabla_{q q} U(q) \succ 0$. Inspection of (10) shows that the matrix $\widehat{M^{(l)}}$ is positive definite for any choice of time-step $h$, and the results of Theorem 2.2 apply to guarantee that the scheme (12)-(16) is well posed.

The second case is possibly the most common case in rigid body dynamics: the case where the stiff forces originate in springs and dampers attached between two points of the multibody system. 
Let $\hat{\phi}(q)$ be a mapping that defines the distance between two points in global coordinates. Then the total energy stored in the spring will be $(\gamma / 2)\left(\hat{\phi}(q)-\hat{\phi}_{0}\right)^{2}$, where $\hat{\phi}_{0}$ is the distance between the points at which the spring is in equilibrium, and $\gamma$ is the spring (elasticity) parameter. To simplify notation, we denote $\phi(q)=\hat{\phi}(q)-\hat{\phi}_{0}$. By applying the fundamental principles of rational mechanics [12], we obtain that the force exerted by the spring on the system is equal to

$$
-\nabla_{q} \frac{\gamma}{2}(\phi(q))^{2}=-\gamma \phi(q) \nabla_{q} \phi(q)
$$

Similarly, the force exerted by a damper attached between two points in the system will be

$$
-\delta\left(\nabla_{q} \phi^{\mathrm{T}}(q) v\right) \nabla_{q} \phi(q)
$$

where $v$ is the velocity of the system and $\delta$ is the damping parameter.

For the general case where there are several springs and dampers between pairs of points of the system, in addition to a non-stiff force, we obtain the following expression for the total external force:

$$
\begin{aligned}
k(t, q, v)= & -\sum_{i=1}^{n_{\gamma}} \gamma_{i} \phi^{(i)}(q) \nabla_{q} \phi^{(i)}(q)-\sum_{j=1}^{n_{\delta}} \delta_{j} \nabla_{q} \psi^{(j)}(q)\left(\nabla_{q} \psi(j)^{\mathrm{T}}(q) v\right) \\
& -\sum_{k=1}^{n_{\delta \gamma}}\left(\bar{\gamma}_{k} \bar{\phi}^{(k)}(q)+\bar{\delta}_{k}\left(\nabla_{q} \bar{\phi}^{(k)^{\mathrm{T}}}(q) v\right)\right) \nabla_{q} \bar{\phi}^{(k)}(q)-k_{\mathrm{c}}(q, v, v)+k_{1}(t, q, v)
\end{aligned}
$$

Here $\gamma_{i}, i=1,2, \ldots, n_{\gamma}$ and $\bar{\gamma}_{k}, k=1,2, \ldots, n_{\delta \gamma}$, are spring (or elastic) parameters, and $\delta_{j}$, $j=1,2, \ldots, n_{\delta}, \bar{\delta}_{k}, k=1,2, \ldots, n_{\gamma \delta}$, are the damping parameters. Here the functions $\phi^{(i)}(q)$, $\psi^{(j)}(q)$, and $\bar{\phi}^{(k)}(q)$ are related to the distances between the points where the springs and the dampers are attached (they can, for example, be distances offset by some constant value). The functions $\bar{\phi}^{(k)}(q), k=1,2, \ldots, n_{\delta \gamma}$, are associated with pairs of points between which there are both springs and dampers. Although a distance function is generally not differentiable everywhere, we will assume that the coordinates of the system will vary in a region where $\phi^{(i)}(q)$ and $\psi^{(j)}(q)$ are differentiable. The distance can also be measured in angular co-ordinates, and our set-up could also accommodate a spring or a damper around the fixed point of a revolute joint, for example.

The quantity $-k_{\mathrm{c}}(q, v, v)$ denote the centrifugal and Coriolis forces, and is a bilinear form in its last two variables. This quantity also depends on the mass tensor. However, that dependence will not influence our analysis, and we do not consider it explicitly here. Since the centrifugal and Coriolis forces are not involved in the energy balance, they must satisfy $k_{\mathrm{c}}(q, v, v)^{\mathrm{T}} v=0$. As we will show with the Newton-Euler formulation in body co-ordinates, $k_{\mathrm{c}}$ can be defined in such a fashion that

$$
k_{\mathrm{c}}\left(q, v_{1}, v_{2}\right)^{\mathrm{T}} v_{2}=0, \quad \forall q, v_{1}, v_{2}
$$

Since the mapping $k_{\mathrm{c}}\left(q, v_{1}, v_{2}\right)$ is linear in $v_{2}$, we can associate to it the matrix $K_{\mathrm{c}}\left(q, v_{1}\right)$, that satisfies

$$
K_{\mathrm{c}}\left(q, v_{1}\right) v_{2}=k_{\mathrm{c}}\left(q, v_{1}, v_{2}\right)
$$


Relation (27) can be rephrased in terms of the matrix $K_{\mathrm{c}}\left(q, v_{1}\right)$ as

$$
v_{2}^{\mathrm{T}} K_{\mathrm{c}}\left(q, v_{1}\right)^{\mathrm{T}} v_{2}=0, \quad \forall q, v_{1}, v_{2}
$$

which ensures that the matrix $K_{\mathrm{c}}\left(q, v_{1}\right)$ is antisymmetric and thus positive semi-definite.

The function $k_{1}(t, q, v)$ cumulates the rest of external forces, which we will assume to be non-stiff, and will be thus treated in an explicit manner.

When setting up the scheme corresponding to (12)-(16), we will work with approximations of the derivatives $\nabla_{q} k(q, v)$ and $\nabla_{v} k(q, v)$. Approximate Jacobians are used in several linearly implicit or Rosenbrock methods [15]. In our case, to define the matrices $\widehat{M^{(l)}}(10)$ and $\widehat{k^{(l)}}$ (11), we will use the following approximations:

$$
\begin{aligned}
& \nabla_{q} k(t, q, v) \approx-\sum_{i=1}^{n_{\gamma}} \gamma_{i} \nabla_{q} \phi^{(i)}(q) \nabla_{q} \phi^{(i)^{\mathrm{T}}}(q)-\sum_{k=1}^{n_{\delta \gamma}} \bar{\gamma}_{k} \nabla_{q} \bar{\phi}^{(k)}(q) \nabla_{q} \bar{\phi}^{(k)^{\mathrm{T}}}(q) \\
& \nabla_{v} k(t, q, v) \approx-\sum_{i=1}^{n_{\delta}} \delta_{j} \nabla_{q} \psi^{(j)}(q) \nabla_{q} \psi^{(j)^{\mathrm{T}}}(q)-\sum_{k=1}^{n_{\delta \gamma}} \bar{\delta}_{k} \nabla_{q} \bar{\phi}^{(k)}(q) \nabla_{q} \bar{\phi}^{(k)^{\mathrm{T}}}(q)
\end{aligned}
$$

Note that the approximation of the gradient $\nabla_{v} k(t, q, v)$ is exact except for the terms appearing in $k_{1}(t, q, v)$.

The term involving $k_{\mathrm{c}}(q, v, v)$ will be treated in a semi-implicit fashion. At step $(l)$ it will enter our scheme as $k_{\mathrm{c}}\left(q^{(l)}, v^{(l)}, v^{(l+1)}\right)=K_{\mathrm{c}}\left(q^{(l)}, v^{(l)}\right) v^{(l+1)}$, the equality being based on (28). A choice of $k_{\mathrm{c}}\left(q^{(l)}, v^{(l)}, v^{(l)}\right)$ corresponds to an explicit Euler method, whereas $k_{\mathrm{c}}\left(q^{(l+1)}, v^{(l+1)}\right.$, $\left.v^{(l+1)}\right)$ corresponds to a fully implicit Euler method (in the absence of all other constraints). As it is the case with explicit and fully implicit Euler choices, our choice does not modify the order of the method.

With the approximations (30) and (31) applied to (10), and (11) we obtain

$$
\begin{aligned}
\widehat{M^{(l)}}= & M\left(q^{(l)}\right)+h K_{\mathrm{c}}\left(q^{(l)}, v^{(l)}\right)+h^{2} \sum_{i=1}^{n_{\gamma}} \gamma_{i} \nabla_{q} \phi^{(i)}\left(q^{(l)}\right) \nabla_{q} \phi^{(i)^{\mathrm{T}}}\left(q^{(l)}\right) \\
& +h \sum_{j=1}^{n_{\delta}} \delta_{j} \nabla_{q} \psi^{(j)}\left(q^{(l)}\right) \nabla_{q} \psi^{(j)^{\mathrm{T}}}\left(q^{(l)}\right)+\sum_{k=1}^{n_{\delta \gamma}}\left(h \bar{\delta}_{k}+h^{2} \bar{\gamma}_{k}\right) \nabla_{q} \bar{\phi}^{(k)}\left(q^{(l)}\right) \nabla_{q} \bar{\phi}^{(k)^{\mathrm{T}}}\left(q^{(l)}\right)
\end{aligned}
$$

and

$$
\widehat{k^{(l)}}=-\sum_{i=1}^{n_{\gamma}} \gamma_{i} \phi^{(i)}\left(q^{(l)}\right) \nabla_{q} \phi^{(i)}\left(q^{(l)}\right)-\sum_{k=1}^{n_{\delta \gamma}} \bar{\gamma}_{k} \bar{\phi}^{(k)}\left(q^{(l)}\right) \nabla_{q} \bar{\phi}^{(k)}\left(q^{(l)}\right)+k_{1}\left(t^{(l)}, q^{(l)}, v^{(l)}\right)
$$

The linearly implicit Euler LCP for a stiff force of the type (26) simply becomes (12)-(16), where the matrices $\widehat{M^{(l)}}$ and $\widehat{k^{(l)}}$ are defined by (32) and (33), respectively. The important feature of our setup is that the matrix $\widehat{M^{(l)}}$ is clearly positive definite, since the mass matrix $M\left(q^{(l)}\right)$ is positive definite, since the matrix $h K_{\mathrm{c}}\left(q^{(l)}, v^{(l)}\right)$ is antisymmetric and thus positive semi-definite, and since terms of the type $a a^{\mathrm{T}}$, where $a$ is an arbitrary column vector, are positive semi-definite matrices. 
In this case Theorem 2.2 applies for any choice of the timestep $h$, showing that the scheme (12)-(16) with the matrix $\widehat{M^{(l)}}$ above has a solution for any choice of the time-step.

\subsection{Stability as stiffness increases to infinity}

One of the desirable properties of stiff schemes is to remain stable as the stiffness parameters go to infinity. This ensures that the scheme will behave well for any choice of the stiffness parameters. We will discuss under what conditions our scheme has the same properties if the stiff force is of the form (26).

2.3.1. The limit system. Intuitively, it is clear that as the stiffness parameters approach infinity, the system should behave as if there were a link (distance constraint) between the points where the damper and/or spring is attached. Therefore, in addition to the original constraints of the system, the system obtained in the stiff limit includes the additional constraints $\phi^{(i)}(q)=0, i=1,2, \ldots, n_{\gamma}, \psi^{(j)}(q)=0, j=1,2, \ldots, n_{\delta}$, and $\bar{\phi}^{(k)}(q)=0, k=1,2, \ldots, n_{\gamma \delta}$. When writing the time-stepping scheme, we replace the constraints with their linearized versions. For example, $\phi^{(i)}(q)=0$ is replaced by $\nabla_{q} \phi^{(i)^{\mathrm{T}}}(q) v=0$, for $i=1,2, \ldots, n_{\gamma}$. Adding such linear equality constraints corresponding to the limit case of the stiff force (26) to (7), we obtain the following time-stepping LCP:

$$
q^{(l+1)}=q^{(l)}+h \bar{v}^{(l+1)}
$$

where $\bar{v}^{(l+1)}$ is a solution of the mixed linear complementarity problem:

$$
\begin{gathered}
\left(M\left(q^{(l)}\right)+h K_{\mathrm{c}}\left(q^{(l)}, v^{(l)}\right)\right) \bar{v}^{(l+1)}-\sum_{i=1}^{m} \bar{v}^{(i)} \bar{c}_{v}^{(i)}-\sum_{j \in \mathscr{A}}\left(n^{(j)} \bar{c}_{n}^{(j)}+D^{(j)} \bar{\beta}^{(j)}\right)-\bar{K}_{s} \\
=h k\left(t, q^{(l)}, v^{(l)}\right)+M\left(q^{(l)}\right) v^{(l)} \\
v^{(i)^{\mathrm{T}}} \bar{v}^{(l+1)}=0, \quad i=1,2, \ldots, m \\
\nabla_{q} \phi^{(i)^{\mathrm{T}}} \bar{v}^{(l+1)}=0, \quad i=1,2, \ldots, n_{\gamma} \\
\nabla_{q} \psi^{(j)^{\mathrm{T}}} \bar{v}^{(l+1)}=0, \quad j=1,2, \ldots, n_{\delta} \\
\nabla_{q} \bar{\phi}^{(k)^{\mathrm{T}}} \bar{v}^{(l+1)}=0, \quad k=1,2, \ldots, n_{\delta \gamma} \\
\bar{\rho}^{(j)}=n^{(j)^{\mathrm{T}}} \bar{v}^{(l+1)} \geqslant 0, \quad \perp \bar{c}_{n}^{(j)} \geqslant 0, \quad j \in \mathscr{A} \\
\bar{\sigma}^{(j)}=\bar{\lambda}^{(j)} e^{(j)}+D^{(j) \mathrm{T}} \bar{v}^{(l+1)} \geqslant 0, \quad \perp \bar{\beta}^{(j)} \geqslant 0, \quad j \in \mathscr{A} \\
\bar{\zeta}^{(j)}=\mu^{(j)} \bar{c}_{n}^{(j)}-e^{(j)^{\mathrm{T}}} \bar{\beta}^{(j)} \geqslant 0, \quad \perp \bar{\lambda}^{(j)} \geqslant 0, \quad j \in \mathscr{A}
\end{gathered}
$$

with

$$
\bar{K}_{s}=\sum_{i=1}^{n_{\gamma}} \bar{c}_{\gamma}^{(i)} \nabla_{q} \phi^{(i)}(q)+\sum_{j=1}^{n_{\delta}} \bar{c}_{\delta}^{(j)} \nabla_{q} \psi^{(j)^{\mathrm{T}}}(q)+\sum_{k=1}^{n_{\delta \gamma}} \bar{c}_{\delta \gamma}^{(k)} \nabla_{q} \bar{\phi}^{(k)}(q)
$$


The linear complementarity problem (34)-(35) will always have a solution, as a result of Theorem 2.2 and positive definiteness of the mass $M\left(q^{(l)}\right)$. We denote a solution of this complementarity problem by

$$
\mathscr{U}=\left(\bar{v}^{(l+1)}, \tilde{\bar{c}}_{v}, \tilde{\bar{c}}_{m}, \tilde{\bar{\beta}}, \tilde{\bar{c}}_{\gamma}, \tilde{\bar{c}}_{\delta}, \tilde{\bar{c}}_{\delta \gamma}\right)
$$

As before, the symbol is used to denote aggregate quantities with the same base symbol, such as $\tilde{\bar{c}}_{\gamma}=\left(\bar{c}_{\gamma}^{(1)}, \bar{c}_{\gamma}^{(2)}, \ldots, \bar{c}_{\gamma}^{\left(n_{\gamma}\right)}\right)$.

2.3.2. Pointedness of the friction cone. To analyze the limit behaviour of (12)-(16), as we let the stiffness parameters from (26) go to infinity, we will assume a certain regular behaviour of the friction cone of the limit system. Anticipating that, we denote by $\Gamma$ the aggregate vector of stiffness parameters:

$$
\Gamma=\left(\gamma_{1}, \gamma_{2}, \ldots, \gamma_{n_{\gamma}}, \delta_{1}, \delta_{2}, \ldots, \delta_{n_{\delta}}, \bar{\gamma}_{1}, \bar{\gamma}_{2}, \ldots, \bar{\gamma}_{n_{\gamma \delta}}, \bar{\delta}_{1}, \bar{\delta}_{2}, \ldots, \bar{\delta}_{n_{\gamma \delta}}\right)
$$

Inspecting the time-stepping LCP (34), we obtain the following expression for the total friction cone of the limit problem:

$$
\begin{aligned}
\widehat{\mathrm{FC}}(q)= & \left\{f \mid f=\sum_{i=1}^{m} \bar{v}^{(i)} \bar{c}_{v}^{(i)}+\sum_{j \in \mathscr{A}}\left(n^{(j)} \bar{c}_{n}^{(j)}+D^{(j)} \bar{\beta}^{(j)}\right)+\sum_{i=1}^{n_{\gamma}} \bar{c}_{\gamma}^{(i)} \nabla_{q} \phi^{(i)}(q)+\sum_{j=1}^{n_{\delta}} \bar{c}_{\delta}^{(j)} \nabla_{q} \psi^{(j)^{\mathrm{T}}}(q)\right. \\
& \left.+\sum_{k=1}^{n_{\delta \gamma}} \bar{c}_{\delta \gamma}^{(k)} \nabla_{q} \bar{\phi}^{(k)}(q) \mid \bar{c}_{n}^{(j)} \geqslant 0, \bar{\beta}^{(j)} \geqslant 0, \mu^{(j)} \bar{c}_{n}^{(j)}-e^{(j)^{\mathrm{T}}} \bar{\beta}^{(j)} \geqslant 0, j \in \mathscr{A}\right\}
\end{aligned}
$$

Indeed, the friction cone subsumes contributions from all the constraint forces. It is evident that the cone $\widehat{\mathrm{FC}}(q)$ has a finite number of generators and, as such, is polyhedral and closed.

We say that a cone $\mathscr{K}$ is pointed if it does not contain a proper vector space, or $\mathscr{K} \cap-\mathscr{K}=\{0\}[3,17]$. Therefore, the point 0 will be a vertex of the cone, since no line containing 0 will be entirely contained in a pointed cone $K$.

\section{Assumption}

We assume that the total friction cone $\widehat{\mathrm{FC}(q)}(38)$ is pointed.

This assumption is essential in ensuring that the limit sequence of solutions, as $h \rightarrow 0$, converges to the solution of a measure differential inclusion [3, 18]. As it can be immediately checked, such a condition implies that at least all of the gradients of the equality constraints must be linearly independent.

If the friction cone is pointed, then it follows immediately, from a duality argument [18] and using the notations in (38) and (36), that there exists a constant $c_{\mathrm{FC}}>0$ such that

$$
f \in \widehat{\mathrm{FC}}(q) \Rightarrow\left\|\left(\tilde{\overline{\bar{c}}}, \tilde{\overline{\bar{c}}}, \tilde{\overline{\bar{\beta}}}, \tilde{\bar{c}}_{\gamma}, \tilde{\bar{c}}_{\delta}, \tilde{\bar{c}}_{\delta \gamma}\right)\right\| \leqslant c_{\mathrm{FC}}\|f\|
$$

Therefore the pointedness of the friction cone ensures that a finite total reaction impulse can be generated only by finite impulses at the constraints: joints, contacts, or friction.

2.3.3. Energy inequalities for the stiff system. To demonstrate the limit behaviour, we first find a bound on the system velocity at the new step, $v^{(l+1)}$. Again, the stiff force under 
discussion is defined in (26), and the time-stepping procedure is defined by (12)-(16), where the matrices $\widehat{M^{(l)}}$ and $\widehat{k^{(l)}}$ are given by (32) and (33).

We start with the following lemma.

Lemma 2.3

Assume that $u, v \in \mathbb{R}^{n}, w_{1}, w_{2}, \ldots, w_{m} \in \mathbb{R}, r_{1}, r_{2}, \ldots, r_{m} \in \mathbb{R}$ and $\beta_{1}, \beta_{2}, \ldots, \beta_{m} \in \mathbb{R}_{+}$satisfy the inequality

$$
\|u\|^{2}+\sum_{i=1}^{m} \beta_{i} w_{i}^{2} \leqslant u^{\mathrm{T}} v-\sum_{i=1}^{m} \beta_{i} w_{i} r_{i}
$$

Then

$$
\|u\|^{2}+\sum_{i=1}^{m} \beta_{i}\left(w_{i}+r_{i}\right)^{2} \leqslant\|v\|^{2}+\sum_{i=1}^{m} \beta_{i} r_{i}^{2}
$$

Proof

Manipulating the assumed inequality and using the identity

$$
a^{2}+a b=a(a+b)=(a+b)^{2}-b(a+b)
$$

we deduce that the variables in the statement of the lemma satisfy the inequality

$$
\|u\|^{2}+\sum_{i=1}^{m} \beta_{i}\left(w_{i}+r_{i}\right)^{2} \leqslant u^{\mathrm{T}} v+\sum_{i=1}^{m} \beta_{i} r_{i}\left(w_{i}+r_{i}\right)
$$

The Cauchy Schwarz inequality implies that

$$
\|u\|^{2}+\|v\|^{2}+\sum_{i=1}^{m} \beta_{i}\left(\left(w_{i}+r_{i}\right)^{2}+r_{i}^{2}\right) \geqslant 2 u^{\mathrm{T}} v+2 \sum_{i=1}^{m} \beta_{i} r_{i}\left(w_{i}+r_{i}\right)
$$

and by using the inequality (40) we get

$$
\begin{aligned}
0 & \leqslant-\left(\|u\|^{2}+\sum_{i=1}^{m} \beta_{i}\left(w_{i}+r_{i}\right)^{2}\right)+u^{\mathrm{T}} v+\sum_{i=1}^{m} \beta_{i}\left(w_{i}+r_{i}\right) r_{i} \\
& \leqslant-\left(u^{\mathrm{T}} v+\sum_{i=1}^{m} \beta_{i}\left(w_{i}+r_{i}\right) r_{i}\right)+\|v\|^{2}+\sum_{i=1}^{m} \beta_{i} r_{i}^{2}
\end{aligned}
$$

After manipulating the last inequalities, we obtain

$$
\|u\|^{2}+\sum_{i=1}^{m} \beta_{i}\left(w_{i}+r_{i}\right)^{2} \leqslant u^{\mathrm{T}} v+\sum_{i=1}^{m} \beta_{i}\left(w_{i}+r_{i}\right) r_{i} \leqslant\|v\|^{2}+\sum_{i=1}^{m} \beta_{i} r_{i}^{2}
$$

which proves the claim.

We now prove our main energy inequality.

Theorem 2.4

Consider the time-stepping linearly implicit Euler scheme (12)-(16), where the matrices $\widehat{M^{(l)}}$ and $\widehat{k^{(l)}}$ are defined by (32) and (33). The velocity solution of (12)-(16) 
satisfies

$$
\begin{aligned}
& v^{(l+1)^{\mathrm{T}}} M\left(q^{(l)}\right) v^{(l+1)^{\mathrm{T}}}+\sum_{i=1}^{n_{\gamma}} \gamma_{i}\left(\phi^{(i)}\left(q^{(l)}\right)+h \nabla_{q} \phi^{(i)^{\mathrm{T}}}\left(q^{(l)}\right) v^{(l+1)}\right)^{2} \\
& +\sum_{k=1}^{n_{\delta \gamma}} \bar{\gamma}_{k}\left(\bar{\phi}^{(k)}\left(q^{(l)}\right)+h \nabla_{q} \bar{\phi}^{(k)^{\mathrm{T}}}\left(q^{(l)}\right) v^{(l+1)}\right)^{2} \leqslant \hat{w}^{\mathrm{T}} M\left(q^{(l)}\right) \hat{w} \\
& +\sum_{i=1}^{n_{\gamma}} \gamma_{i}\left(\phi^{(i)}\left(q^{(l)}\right)\right)^{2}+\sum_{k=1}^{n_{\delta \gamma}} \bar{\gamma}_{k}\left(\bar{\phi}^{(k)}\left(q^{(l)}\right)\right)^{2}
\end{aligned}
$$

where $\hat{w}=\left(v^{(l)}+k h M\left(q^{(l)}\right)^{-1} k_{1}\left(t^{(l)}, q^{(l)}, v^{(l)}\right)\right)^{\mathrm{T}}$.

Proof

Left multiplying $(12)$ by $v^{(l+1)^{\mathrm{T}}}$ we get that

$$
\begin{aligned}
v^{(l+1)^{\mathrm{T}}} \widehat{M^{(l)}} v^{(l+1)}= & \sum_{i=1}^{m}\left(v^{(i)^{\mathrm{T}}} v^{(l+1)}\right) c_{v}^{(i)}+\sum_{j \in \mathscr{A}}\left(n^{(j)^{\mathrm{T}}} v^{(l+1)}\right) c_{n}^{(j)}+D^{(j)^{\mathrm{T}}} v^{(l+1)} \beta^{(j)} \\
& +h \widehat{k^{(l)^{\mathrm{T}}}} v^{(l+1)}+v^{(l+1)^{\mathrm{T}}} M\left(q^{(l)}\right) v^{(l)}
\end{aligned}
$$

Using (13), we deduce that $v^{(i)^{\mathrm{T}}} v^{(l+1)}=0, i=1,2, \ldots, m$. Also, using the contact constraints (14), we obtain $n^{(j)^{\mathrm{T}}} v^{(l+1)} c_{n}^{(j)}=0, j \in \mathscr{A}$. Finally, from the frictional constraints (15) and (16) we get that

$$
D^{(j)^{\mathrm{T}}} v^{(l+1)} \beta^{(j)}=-\lambda^{(j)} \beta^{(j)^{\mathrm{T}}} e^{(j)}=-\mu^{(j)} c_{n}^{(j)} \lambda^{(j)} \leqslant 0, \quad \forall j \in \mathscr{A}
$$

Then (41) implies

$$
v^{(l+1)^{\mathrm{T}}} \widehat{M^{(l)}} v^{l+1} \leqslant h \widehat{k^{(l)^{\mathrm{T}}}} v^{(l+1)}+v^{(l+1)^{\mathrm{T}}} M\left(q^{(l)}\right) v^{(l)}
$$

We now use the definitions (32) of $\widehat{M^{(l)}}$ and (33) of $\widehat{k^{(l)}}$, as well as the fact that $v^{(l+1)^{\mathrm{T}}} K_{\mathrm{c}}\left(q^{(l)}\right.$, $\left.v^{(l)}\right) v^{(l+1)}=0$ from (29), to obtain that

$$
\begin{aligned}
v^{(l+1)^{\mathrm{T}}} \widehat{M^{(l)}} v^{(l+1)}= & v^{(l+1)^{\mathrm{T}}} M\left(q^{(l)}\right) v^{(l+1)}+h^{2} \sum_{i=1}^{n_{\gamma}} \gamma_{i}\left(\nabla_{q} \phi^{(i)^{\mathrm{T}}}\left(q^{(l)}\right) v^{(l+1)}\right)^{2} \\
& +h \sum_{j=1}^{n_{\delta}} \delta_{j}\left(\nabla_{q} \psi^{(j)^{\mathrm{T}}}\left(q^{(l)}\right) v^{(l+1)}\right)^{2}+\sum_{k=1}^{n_{\delta \gamma}}\left(h \bar{\delta}_{k}+h^{2} \bar{\gamma}_{k}\right)\left(\nabla_{q} \bar{\phi}^{(k)^{\mathrm{T}}}\left(q^{(l)}\right) v^{(l+1)}\right)^{2} \\
\geqslant & v^{(l+1)^{\mathrm{T}}} M\left(q^{(l)}\right) v^{(l+1)}+h^{2} \sum_{i=1}^{n_{\gamma}} \gamma_{i}\left(\nabla_{q} \phi^{(i)^{\mathrm{T}}}\left(q^{(l)}\right) v^{(l+1)}\right)^{2} \\
& +\sum_{k=1}^{n_{\delta \gamma}} h^{2} \bar{\gamma}_{k}\left(\nabla_{q} \bar{\phi}^{(k)^{\mathrm{T}}}\left(q^{(l)}\right) v^{(l+1)}\right)^{2}
\end{aligned}
$$


and, respectively,

$$
\begin{aligned}
h v^{(l+1)^{\mathrm{T}} \widehat{k^{(l)}}=} & -\sum_{i=1}^{n_{\gamma}} \gamma_{i} \phi^{(i)}\left(q^{(l)}\right)\left(h \nabla_{q} \phi^{(i)}\left(q^{(l)}\right)^{\mathrm{T}} v^{(l+1)}\right) \\
& -\sum_{k=1}^{n_{\delta \gamma}} \bar{\gamma}_{k} \bar{\phi}^{(k)}\left(q^{(l)}\right)\left(h \nabla_{q} \bar{\phi}^{(k)}\left(q^{(l)}\right)^{\mathrm{T}} v^{(l+1)}\right)+h k_{1}\left(t^{(l)}, q^{(l)}, v^{(l)}\right)^{\mathrm{T}} v^{(l+1)}
\end{aligned}
$$

Denoting $u=M\left(q^{(l)}\right)^{1 / 2} v^{(l+1)}$ and $w=M\left(q^{(l)}\right)^{1 / 2} v^{(l+1)}+h M\left(q^{(l)}\right)^{-1 / 2} k_{1}\left(t^{(l)}, q^{(l)}, v^{(l)}\right)$, and using the inequalities (43) and (44) in (42), we obtain

$$
\begin{aligned}
& u^{\mathrm{T}} u+\sum_{i=1}^{n_{\gamma}} \gamma_{i}\left(h \nabla_{q} \phi^{(i)^{\mathrm{T}}}\left(q^{(l)}\right) v^{(l+1)}\right)^{2}+\sum_{k=1}^{n_{\delta \gamma}} \bar{\gamma}_{k}\left(h \nabla_{q} \bar{\phi}^{(k)^{\mathrm{T}}}\left(q^{(l)}\right) v^{(l+1)}\right)^{2} \\
& \geqslant u^{\mathrm{T}} w-\sum_{i=1}^{n_{\gamma}} \gamma_{i} \phi^{(i)}\left(q^{(l)}\right)\left(h \nabla_{q} \phi^{(i)}\left(q^{(l)}\right)^{\mathrm{T}} v^{(l+1)}\right)-\sum_{k=1}^{n_{\delta \gamma}} \bar{\gamma}_{k} \bar{\phi}^{(k)}\left(q^{(l)}\right)\left(h \nabla_{q} \bar{\phi}^{(k)}\left(q^{(l)}\right)^{\mathrm{T}} v^{(l+1)}\right)
\end{aligned}
$$

We now use Lemma 2.3 to get

$$
\begin{gathered}
u^{\mathrm{T}} u+\sum_{i=1}^{n_{\gamma}} \gamma_{i}\left(\phi^{(i)^{\mathrm{T}}}\left(q^{(l)}\right)+h \nabla_{q} \phi^{(i)^{\mathrm{T}}}\left(q^{(l)}\right) v^{(l+1)}\right)^{2}+\sum_{k=1}^{n_{\delta \gamma}} \bar{\gamma}_{k}\left(\bar{\phi}^{(k)^{\mathrm{T}}}\left(q^{(l)}\right)+h \nabla_{q} \bar{\phi}^{(k)^{\mathrm{T}}}\left(q^{(l)}\right) v^{(l+1)}\right)^{2} \\
\geqslant w^{\mathrm{T}} w+\sum_{i=1}^{n_{\gamma}} \gamma_{i}\left(\phi^{(i)}\left(q^{(l)}\right)\right)^{2}+\sum_{k=1}^{n_{\delta \gamma}} \bar{\gamma}_{k}\left(\bar{\phi}^{(k)}\left(q^{(l)}\right)\right)^{2}
\end{gathered}
$$

By replacing the definitions of $u$ and $w$ we obtain the claim, since $u^{\mathrm{T}} u=v^{(l+1)^{\mathrm{T}}} M\left(q^{(l)}\right) v^{(l+1)^{\mathrm{T}}}$ and $w^{\mathrm{T}} w=\left(v^{(l)}+k h M\left(q^{(l)}\right)^{-1} k_{1}\left(t^{(l)}, q^{(l)}, v^{(l)}\right)\right)^{\mathrm{T}} M\left(q^{(l)}\right)\left(v^{(l)}+k h M\left(q^{(l)}\right)^{-1} k_{1}\left(t^{(l)}, q^{(l)}, v^{(l)}\right)\right)^{\mathrm{T}}$.

When all the springs start from the rest position, we have a special case of this result.

\section{Corollary 2.5}

Assume that $q^{(l)}$ is a point where the springs in the stiff force (26) are at equilibrium, or $\phi^{(i)}(q)=0, i=1,2, \ldots, n_{\gamma}, \bar{\phi}^{(k)}(q)=0, k=1,2, \ldots, n_{\delta \gamma}$. Then the velocity at the new step $v^{(l+1)}$ is bounded uniformly with respect to the stiffness parameters, and the kinetic energy satisfies the inequality

$$
v^{(l+1)^{\mathrm{T}}} M\left(q^{(l)}\right) v^{(l+1)^{\mathrm{T}}} \leqslant \hat{w} M\left(q^{(l)}\right) \hat{w}
$$

where $\hat{w}=\left(v^{(l)}+k h M\left(q^{(l)}\right)^{-1} k_{1}\left(t^{(l)}, q^{(l)}, v^{(l)}\right)\right)^{\mathrm{T}}$.

Proof

The proof follows immediately by replacing $\phi^{(i)}(q)=0, i=1,2, \ldots, n_{\gamma}, \bar{\phi}^{(k)}(q)=0, k=1,2, \ldots$, $n_{\delta \gamma}$ in the conclusion of Theorem 2.4.

The conclusions of Theorem 2.4 and Lemma 2.5 are particularly helpful in the situation where the stiffness in (26) originates in damping-type forces or where the elastic forces are small and can be included in the non-stiff part $k(t, q, l)$. This point can be immediately seen because none of the velocity and kinetic energy bounds depend on the damping 
parameters $\delta_{j}, i=1,2, \ldots, n_{\delta}$ and $\bar{\delta}_{k}, k=1,2, \ldots, n_{\gamma \delta}$. The lack of an immediate similar extension for large elastic forces is to be expected, because if we increase the values of the elasticity parameters, then the total energy will increase. Therefore, we cannot expect that the kinetic energy will stay bounded if we increase the elastic parameter when the spring is not at equilibrium.

However, under the following assumptions an even stronger result can be obtained:

- The mass matrix $M\left(q^{(l)}\right)$ is constant. This is true for the case where the system is parameterized in the Newton-Euler body co-ordinates [11].

- The mappings $\phi^{(i)}(q), i=1,2, \ldots, n_{\gamma}, \bar{\phi}^{(j)}(q), j=1,2, \ldots, n_{\delta \gamma}$, are linear.

In that case, the left-hand side of the inequality from the conclusion of Theorem 2.4 represents twice the total energy at $\left(q^{(l+1)}, v^{(l+1)}\right)$. Therefore Theorem 2.4 can be applied in conjunction with Theorem 5.1 in Reference [7] to show that the total energy remains uniformly bounded with respect to the time step $h$ for any fixed time interval as soon as the function $k_{1}(t, q, v)$ is bounded.

In spite of the fact that few mechanical systems satisfy the second requirement, our analysis and conclusions are consistent with the general set-up of stability analysis, where stability of stiff systems is evaluated on the linearized system [15].

Although Corollary 2.5 applies to a very particular case, it does suggest that the method will be likely to behave well even for very large values of the elasticity parameters in a neighbourhood of the equilibrium position of the springs. However, we cannot guarantee that the total energy of the system will remain within a bound that depends only on its initial value and the size of the non-stiff force $k_{1}$. In general, this can be ensured essentially only for symplectic methods [15]. Because of friction, our system is not Hamiltonian; and as such, the symplecticity concept does not apply, even if we were willing to solve a nonlinear equation for $v^{(l+1)}$.

2.3.4. Controlling the energy behaviour and constraint infeasibility by projection. Consider again the time-stepping linearly implicit Euler scheme $q^{(l+1)}=q^{(l)}+h v^{(l+1)}$, where $v^{(l+1)}$ is a solution of (12)-(16), with the matrices $\widehat{M^{(l)}}$ and $\widehat{k^{(l)}}$ are defined by (32) and (33).

Owing to the fact that the joint (1) and non-interpenetration constraints (2)

$$
\Theta^{(i)}(q)=0, \quad i=1,2, \ldots, m ; \quad \Phi^{(j)}(q) \geqslant 0, \quad j=1,2, \ldots, p
$$

are generally nonlinear mappings, $q^{(l+1)}$ will not satisfy these constraint exactly. Nevertheless, due to the semi-implicit nature of our time-stepping scheme, the velocities satisfy the linearized geometrical constraints exactly. It has been shown that, for most numerical schemes that solve differential algebraic equations, this property is sufficient to ensure constraint stability for fairly long simulation intervals [15, Section VII.2].

If, however, the error in satisfying the constraints (45), is too large to be tolerated, then one can apply a projection towards the feasible set of (45). In the presence of stiffness due to large spring parameters in the stiff force (26), such a projection may interfere with our energy bound from Theorem 2.4.

To accommodate this problem, we may use the following constraint stabilization strategy, whenever constraint violation is deemed to be excessive:

(1) $\tilde{q}^{(l+1)}=q^{(l)}+h v^{(l+1)}$, where $v^{(l+1)}$ is a solution of $(12)-(16)$, with the matrices $\widehat{M^{(l)}}$ and $\widehat{k^{(l)}}$ are defined by (32) and (33). 
(2) Determine the next position vector $q^{(l+1)}$, the solution of the following nonlinear projection problem:

$$
\begin{array}{cl}
\min _{q} & \left\|q-\tilde{q}^{(l+1)}\right\|^{2} \\
\text { such that } & \Theta^{(i)}(q)=0, \quad i=1,2, \ldots, m \\
& \Phi^{(j)}(q) \geqslant 0, \quad j=1,2, \ldots, p \\
& \phi^{\left(i_{1}\right)}(q)=\phi^{\left(i_{1}\right)}\left(q^{(l)}\right)+h \nabla_{q} \phi^{\left(i_{1}\right)^{\mathrm{T}}}\left(q^{(l)}\right) v^{(l+1)}, \quad i_{1}=1,2, \ldots, n_{\gamma} \\
& \bar{\phi}^{(k)}(q)=\bar{\phi}^{(k)}\left(q^{(l)}\right)+h \nabla_{q} \bar{\phi}^{(k)^{\mathrm{T}}}\left(q^{(l)}\right) v^{(l+1)}, \quad k=1,2, \ldots, n_{\delta \gamma}
\end{array}
$$

Under the assumption that the friction cone of the limit system as the stiffness goes to infinity is pointed, which is detailed in SubSection 2.4, it can be immediately shown that the nonlinear program (46) satisfies the Mangasarian-Fromovitz constraint qualification (the set defined by replacing the constraints in (46) by their linearization has a non-empty interior relative to the inequality constraints, [19]). This $q^{(l+1)}$ can be obtained by a sequential quadratic programming algorithm [23]. Under the same conditions it can be also shown that the solution set of (46) is Lipschitz continuous with respect to perturbations [19]. If the infeasibility in the constraints (45) does not exceed $O\left(h^{2}\right)$, then it can be shown that $\left\|q^{(l+1)}-\tilde{q}^{(l+1)}\right\|=O\left(h^{2}\right)$ if the velocity $v^{(l+1)}$ is bounded. Therefore the projection does not alter the order of the method. Under the stronger assumption that the gradients of the equality constraints and potentially active inequality constraints in (46) are linearly independent, we can replace the inequality constraints by $\Phi^{(j)}(q)=0, j \in \mathscr{A}$, where $\mathscr{A}$ is the set of indices of constraints that are currently active. However, such assumptions are too strong for some simple configurations, such as a rectangular block on a tabletop [13].

After the position vector is projected onto the feasible set thus obtaining the new position $q^{(l+1)}$, the velocities need not be readapted since our semi-implicit formulation ensures velocity consistency when computing $\tilde{q}^{(l+2)}$.

After the use of a projection, we can write the following energy bound, using that $q^{(l+1)}$ satisfies the constraints of (46) and the conclusion of Theorem 2.4, which now applies to $\tilde{q}^{(l+1)}$ :

$$
\begin{gathered}
v^{(l+1)^{\mathrm{T}}} M\left(q^{(l)}\right) v^{(l+1)^{\mathrm{T}}}+\sum_{i=1}^{n_{\gamma}} \gamma_{i}\left(\phi^{(i)}\left(q^{(l+1)}\right)\right)^{2}+\sum_{k=1}^{n_{\delta \gamma}} \bar{\gamma}_{k}\left(\bar{\phi}^{(k)}\left(q^{(l+1)}\right)\right)^{2} \\
\geqslant \hat{w}^{\mathrm{T}} M\left(q^{(l)}\right) \hat{w}+\sum_{i=1}^{n_{\gamma}} \gamma_{i}\left(\phi^{(i)}\left(q^{(l)}\right)\right)^{2}+\sum_{k=1}^{n_{\delta \gamma}} \bar{\gamma}_{k}\left(\bar{\phi}^{(k)}\left(q^{(l)}\right)\right)^{2}
\end{gathered}
$$

Here $\hat{w}$ is defined in Theorem 2.4, and we have $\hat{w}=v^{(l)}$ if the external non-stiff force $k_{1}(t, q, v)=0$. It can thus be seen that, if $M\left(q^{(l)}\right)$ is constant, as it is the case for the Newton-Euler equations in body co-ordinates and if the external non-stiff force is 0 then the total energy is decreasing. If $M\left(q^{(l)}\right)$ is constant, and the outside stiff force is uniformly bounded, then the technique from [7] can be applied to show that the total energy cannot increase faster than linearly in time for sufficiently small time step $h$ (same as it happens if a ball is dropped under the effect of gravity before colliding). Therefore the scheme preserves its good energy properties if a projection of the type (46) is used for constraint stabilization. 
Effect of projection when only damping forces act between bodies: In this situation, the use of a projection does not, in effect, alter the energy bound from Theorem 2.4, since velocities are unaffected by the position projection. In that case the nonlinear program describing the projection (46) does not include the last two sets of constraints that appear only when springs are attached and it reduces to the usual projection procedure.

2.3.5. The Newton-Euler equations in body co-ordinates. For one body in a three-dimensional space, the Newton-Euler equations in body co-ordinates are [1]:

$$
\left[\begin{array}{cc}
m I & 0 \\
0 & \mathscr{I}
\end{array}\right]\left[\begin{array}{c}
\dot{v}_{\mathrm{b}} \\
\dot{\omega}_{\mathrm{b}}
\end{array}\right]+\left[\begin{array}{c}
-m v^{\mathrm{b}} \times \omega^{\mathrm{b}} \\
\omega^{\mathrm{b}} \times \mathscr{I} \omega^{\mathrm{b}}
\end{array}\right]=F^{\mathrm{b}}
$$

Here $v^{\mathrm{b}}$ and $\omega^{\mathrm{b}}$ are the translational and, respectively, the rotational velocity in body coordinates, $m$ is the mass of the object, $\mathscr{I}$ is the inertia matrix which is positive definite and constant, and may even be diagonal for the appropriate choice of rotational co-ordinates. Also, $F^{\mathrm{b}}$ is the external force acting on the body. The second vector in the left-hand side of (48) has as components the centrifugal and Coriolis force, respectively.

The generalized velocity becomes $v=\left[\left(v^{\mathrm{b}}\right)^{\mathrm{T}}\left(\omega^{\mathrm{b}}\right)^{\mathrm{T}}\right]^{\mathrm{T}}$. We define the following mapping:

$$
k_{\mathrm{c}}\left(q, v_{1}, v_{2}\right)=\left[\begin{array}{c}
-m v_{2}^{\mathrm{b}} \times \omega_{1}^{\mathrm{b}} \\
\omega_{2}^{\mathrm{b}} \times \mathscr{I} \omega_{1}^{\mathrm{b}}
\end{array}\right]
$$

where $v_{i}=\left[\left(v_{i}^{\mathrm{b}}\right)^{\mathrm{T}}\left(\omega_{i}^{\mathrm{b}}\right)^{\mathrm{T}}\right]^{\mathrm{T}}$, for $i=1,2$. It is immediate from its definition that $k_{\mathrm{c}}\left(q, v_{1}, v_{2}\right)$ is a bilinear map in its last two arguments and that, from the properties of the cross product, it satisfies $v_{2}^{\mathrm{T}} k_{\mathrm{c}}\left(q, v_{1}, v_{2}\right)=0, \forall q, v_{1}, v_{2}$ (it actually does not depend on $q$ in this formulation). From (48) it can also be seen that the inertial and Coriolis forces can be now expressed as $-k_{\mathrm{c}}(q, v, v)$. This shows that our assumptions concerning the inertial and Coriolis forces leading to Equations (27), (28) and (29) are justified in the Newton-Euler body co-ordinates setup.

The mass matrix in these coordinates,

$$
M\left(q^{(l)}\right)=\left[\begin{array}{cc}
m I & 0 \\
0 & \mathscr{I}
\end{array}\right]
$$

does not depend on $q^{(l)}$ and it can be therefore considered constant during time-stepping. For multiple bodies $M\left(q^{(l)}\right)$ is a block diagonal matrix whose blocks are the mass matrices for one body. Similarly the mapping $k_{\mathrm{c}}\left(q, v_{1}, v_{2}\right)$ is constructed by adjoining the $k_{\mathrm{c}}$ maps corresponding to one body. It is immediate that all properties relevant to the discussion in this subsection are preserved.

Our treatment of centrifugal and Coriolis forces is important only in three dimensions. In one and two dimensions, the mass matrix is diagonal and constant in other coordinates as well [1].

Stability of the numerical scheme with respect to mass parameters: Although our main concern is spring and damper or similar type of stiffness, a legitimate question is what happens when some of the mass parameters involved in the configuration are much larger than others, which induces another type of stiffness. 
Inspecting the bound (47) we observe that, if the projection proposed in the previous section is used, for the Newton-Euler set-up in body co-ordinates we can guarantee exact decrease of the total energy, independent of the values of the mass matrix. If no elastic forces act between bodies, then even without the projection the same conclusion can be inferred from Theorem 2.4. This shows that under these assumptions the algorithm is stable with respect to mass stiffness, since the mass parameters cannot alter the energy decrease if the Newton-Euler body set-up is used. The energy bounds show that heavy bodies will move slowly during the simulation if the total energy is small in the beginning.

\subsection{A stability result}

We now analyse the accumulation points of the solution of (12)-(16) as we keep the stepsize $h$ constant and as we increase the stiffness parameters to infinity. We will show that under conditions that ensure that our energy results from the preceding subsection apply, such accumulation points will be solutions $\mathscr{U}$ of (34)-(35). In other words, in the limit, our linearly implicit LCP scheme will behave as would a similar scheme applied to a system with additional joint constraints in place of the dampers and springs.

\section{Theorem 2.6}

Assume that the total friction cone $\widehat{\mathrm{FC}}(q)$ (38) of the limit LCP integration step (34) is pointed. Let $q^{(l)}$ be a position vector point where $\phi^{(i)}(q)=0, i=1,2, \ldots, n_{\gamma}$, and $\phi^{(k)}(q)=0$, $k=1,2, \ldots, n_{\delta \gamma}$. Let $\mathscr{U}^{\Gamma}=\left(v^{(l+1)^{\mathrm{T}}}, \tilde{c}_{v}^{\Gamma}, \tilde{c}_{n}^{\Gamma}, \tilde{\beta}^{\Gamma}, \tilde{\lambda}^{\Gamma}\right)$ be a solution of (17)-(18) where the external force is defined by (26) and the matrices $\widehat{M^{(l)}}$ and $\widehat{k}^{l}$ are defined by (32) and (33), for a particular choice of the stiffness parameters $\Gamma$ (37). Let $\Gamma_{n}$ be a sequence of stiffness parameters such that, as $n \rightarrow \infty$, all its components increase toward infinity. Then the sequence $\mathscr{U}^{\Gamma_{n}}$ is uniformly bounded, and any limit point, together with appropriate multipliers for the additional joint constraints, is a solution of (34).

\section{Proof}

Because we assume that springs are at equilibrium, we obtain from immediate inspection of (33) that $\hat{k}^{(l)}=k\left(t^{(l)}, q^{(l)}, v^{(l)}\right)$.

We introduce the following notations:

$$
\begin{aligned}
\tilde{c}_{\gamma}^{\Gamma}= & h^{2}\left(\gamma_{1} \nabla_{q} \phi^{(1)^{\mathrm{T}}}\left(q^{(l)}\right) v^{(l+1)^{\Gamma}}, \gamma_{2} \nabla_{q} \phi^{(2)^{\mathrm{T}}}\left(q^{(l)}\right) v^{(l+1)^{\Gamma}}, \ldots, \gamma_{n_{\gamma}} \nabla_{q} \phi^{\left(n_{\gamma}\right)^{\mathrm{T}}}\left(q^{(l)}\right) v^{(l+1)^{\mathrm{\Gamma}}}\right) \\
\tilde{c}_{\delta}^{\Gamma}= & h\left(\delta_{1} \nabla_{q} \psi^{(1)^{\mathrm{T}}}\left(q^{(l)}\right) v^{(l+1)^{\Gamma}}, \gamma_{2} \nabla_{q} \psi^{(2)^{\mathrm{T}}}\left(q^{(l)}\right) v^{(l+1)^{\Gamma}}, \ldots, \delta_{n_{\delta}} \nabla_{q} \psi^{\left(n_{\delta}\right)^{\mathrm{T}}}\left(q^{(l)}\right) v^{(l+1)^{\Gamma}}\right) \\
\tilde{c}_{\delta \gamma}^{\Gamma}= & h\left(\left(\bar{\gamma}_{1}+h \bar{\delta}_{1}\right) \nabla_{q} \bar{\phi}^{(1)^{\mathrm{T}}}\left(q^{(l)}\right) v^{(l+1)^{\Gamma}},\left(\bar{\gamma}_{2}+h \bar{\delta}_{2}\right) \nabla_{q} \bar{\phi}^{(2)^{\mathrm{T}}}\left(q^{(l)}\right) v^{(l+1)^{\Gamma}}, \ldots,\right. \\
& \left.\left(\bar{\gamma}_{n_{\delta \gamma}}+h \bar{\delta}_{n_{\delta \gamma}}\right) \nabla_{q} \phi^{\left(n_{\delta \gamma}\right)^{\mathrm{T}}}\left(q^{(l)}\right) v^{(l+1)^{\mathrm{\Gamma}}}\right) \\
\tilde{v}_{\gamma}= & \left(\nabla_{q} \phi^{(1)}\left(q^{(l)}\right), \nabla_{q} \phi^{(2)}\left(q^{(l)}\right), \ldots, \nabla_{q} \phi^{\left(n_{\gamma}\right)}\left(q^{(l)}\right)\right) \\
\tilde{v}_{\delta}= & \left(\nabla_{q} \psi^{(1)}\left(q^{(l)}\right), \nabla_{q} \psi^{(2)}\left(q^{(l)}\right), \ldots, \nabla_{q} \psi^{\left(n_{\delta}\right)}\left(q^{(l)}\right)\right) \\
\tilde{v}_{\gamma \delta}= & \left(\nabla_{q} \bar{\phi}^{(1)}\left(q^{(l)}\right), \nabla_{q} \bar{\phi}^{(2)}\left(q^{(l)}\right), \ldots, \nabla_{q} \bar{\phi}^{\left(n_{\gamma \delta}\right)}\left(q^{(l)}\right)\right)
\end{aligned}
$$


Using the fact that $\mathscr{U}^{\Gamma}$ satisfies (17), the assumption of the theorem, the definitions (32), and the above observation for $\widehat{k^{(l)}}$, we obtain

$$
M\left(q^{l}\right) v^{(l+1)^{\Gamma}}-\tilde{v} \tilde{c}_{v}^{\Gamma}-\tilde{n} \tilde{c}_{n}^{\Gamma}-\tilde{D} \tilde{\beta}^{\Gamma}-\tilde{v}_{\gamma} \tilde{c}_{\gamma}^{\Gamma}-\tilde{v}_{\delta} \tilde{c}_{\delta}^{\Gamma}-\tilde{v}_{\gamma \delta} \tilde{c}_{\gamma \delta}^{\Gamma}=M\left(q^{(l)}\right) v^{(l)}+h k_{1}\left(t^{(l)}, q^{(l)}, v^{(l)}\right)
$$

$\mathscr{U}^{\Gamma}$ is a solution of (12)-(16), and thus satisfies (15) and (16). Hence, from the definition of the friction cone, $\widehat{\mathrm{FC}}\left(q^{(l)}\right)(38)$, we deduce that

$$
M\left(q^{(l)}\right) v^{(l+1)^{\Gamma}}-M\left(q^{(l)}\right) v^{(l)}-h k_{1}\left(t^{(l)}, q^{(l)}, v^{(l)}\right) \in \widehat{\mathrm{FC}}\left(q^{(l)}\right)
$$

Corollary 2.5 implies that $v^{(l+1) \Gamma}$ is bounded uniformly with respect to $\Gamma$, and thus there exists a constant $K_{\mathrm{FC}}$ independent of $\Gamma$ such that

$$
\left\|M\left(q^{l}\right) v^{(l+1)^{\Gamma}}-M\left(q^{(l)}\right) v^{(l)}-h k_{1}\left(t^{(l)}, q^{(l)}, v^{(l)}\right)\right\| \leqslant K_{\mathrm{FC}}
$$

Using now the characterization (39) of the pointed cone $\widehat{\mathrm{FC}}\left(q^{(l)}\right)$, we obtain that, for all $\Gamma$,

$$
\left\|\left(\tilde{c}_{v}^{\Gamma}, \tilde{c}_{n}^{\Gamma}, \tilde{\beta}^{\Gamma}, \tilde{c}_{\gamma}^{\Gamma}, \tilde{c}_{\delta}^{\Gamma}, \tilde{c}_{\gamma \delta}^{\Gamma}\right)\right\| \leqslant c_{\mathrm{FC}} K_{\mathrm{FC}}
$$

Now take the sequence $\Gamma_{n}$. From the previous inequality we deduce that the sequence

$$
\mathscr{W}^{n}=\left(v^{(l+1)^{\Gamma_{n}}}, \tilde{c}_{v}^{\Gamma_{n}}, \tilde{c}_{n}^{\Gamma_{n}}, \tilde{\beta}^{\Gamma_{n}}, \tilde{c}_{\gamma}^{\Gamma_{n}}, \tilde{c}_{\delta}^{\Gamma_{n}}, \tilde{c}_{\gamma \delta}^{\Gamma_{n}}\right)
$$

is uniformly bounded with respect to the sequence $\Gamma_{n}$. Also, following the definition of $\tilde{c}_{\gamma}^{\Gamma}, \tilde{c}_{\delta}^{\Gamma}, \tilde{c}_{\gamma \delta}^{\Gamma}$, and (49), we obtain

$$
\begin{aligned}
\left.h^{2} \gamma_{i}^{n} \mid \nabla_{q} \phi^{(i)}\left(q^{(l)^{\mathrm{T}}}\right) v^{(l+1)^{\mathrm{I}_{n}}}\right) \mid & \leqslant c_{\mathrm{FC}} K_{\mathrm{FC}}, \quad i=1,2, \ldots, n_{\gamma} \\
\left.h \delta_{j}^{n} \mid \nabla_{q} \psi^{(j)}\left(q^{(l)^{\mathrm{T}}}\right) v^{(l+1)^{\mathrm{I}_{n}}}\right) \mid & \leqslant c_{\mathrm{FC}} K_{\mathrm{FC}}, \quad j=1,2, \ldots, n_{\delta} \\
\left.h\left(\bar{\gamma}_{k}^{n}+h \bar{\delta}_{k}^{n}\right) \mid \nabla_{q} \bar{\phi}^{(k)}\left(q^{(l)^{\mathrm{T}}}\right) v^{(l+1)^{\mathrm{I}_{n}}}\right) \mid & \leqslant c_{\mathrm{FC}} K_{\mathrm{FC}}, \quad k=1,2, \ldots, n_{\gamma \delta}
\end{aligned}
$$

Since the sequence $\mathscr{W}^{n}$ is uniformly bounded with respect to $n$, it will have an accumulation point. Let

$$
\overline{\mathscr{W}}=\left(\bar{v}^{(l+1)}, \tilde{\bar{c}}_{v}, \tilde{\bar{c}}, \tilde{\bar{\beta}}, \tilde{\bar{c}}_{\gamma}, \tilde{\bar{c}}_{\delta}, \tilde{\bar{c}}_{\gamma \delta}\right)
$$

be such an accumulation point. For purposes of this proof we will assume, without loss of generality that $\lim _{n \rightarrow \infty} \mathscr{W}^{n}=\overline{\mathscr{W}}$. In particular, we must have that $\lim _{n \rightarrow \infty} v^{(l+1)^{n}}=\bar{v}^{(l+1)}$. From our assumptions, all components of $\Gamma^{n}$ increase to infinity. Dividing (50) with the components of $\Gamma^{n}$ and taking the limit as $n \rightarrow \infty$, we obtain that

$$
\begin{aligned}
& \nabla_{q} \phi^{(i)}\left(q^{(l)^{\mathrm{T}}}\right) \bar{v}^{(l+1)}=0, i=1,2, \ldots, n_{\gamma} \\
& \nabla_{q} \psi^{(j)}\left(q^{(l)^{\mathrm{T}}}\right) \bar{v}^{(l+1)}=0, j=1,2, \ldots, n_{\delta} \\
& \nabla_{q} \bar{\phi}^{(k)}\left(q^{(l)^{\mathrm{T}}}\right) \bar{v}^{(l+1)}=0, \quad k=1,2, \ldots, n_{\gamma \delta}
\end{aligned}
$$

Since $\mathscr{W}^{n}$ satisfies all inequalities and complementarity relations of (17)-(18), which are homogeneous, so will $\overline{\mathscr{W}}$. Since, in addition, $\bar{v}^{(l+1)}$ satisfies $(51)$, we infer that $\overline{\mathscr{W}}$ is indeed a solution of (34), which proves the claim. 


\section{NUMERICAL SIMULATIONS}

To validate the above concepts, we have implemented a Matlab version of the time-stepping procedures described in this work. An important part of any such implementation is to design an appropriate collision resolution strategy. In Reference [7] we presented a method to accommodate a partially elastic collision approach. In the case of rigid bodies, collisions are instantaneous events, where the velocity generally exhibits a discontinuity. The time-stepping procedure must be stopped at the edge of an interpenetration configuration, and a new feasible velocity is found by a collision resolution consistent with the dynamics and geometry of the problem. Since in this work we are interested in simulating very stiff mechanical systems for moderate and relatively large values of the time-step $h$, stiffness treatment is irrelevant during collision.

To simplify the presentation, we assume that all the collisions are purely inelastic $[3,7]$, although partially elastic collisions can also be easily accommodated by a compression-decompression scheme [7]. The inelastic collisions can be incorporated in our approach without any major changes, by taking $h=0$ in $(17)-(18)$, after $t^{(l)}, q^{(l)}$, and $v^{(l)}$ have been determined as the collision data $[7,8]$. In that case $v^{(l+1)}$ simply becomes the postcollision velocity, and the scheme is started at that point. Our previous point is also validated, since for $h=0$ (17) $-(18)$ and (7) are identical LCPs.

As mentioned in Section 2.3.4 we use a projection approach to ensure that the joint constraints and the theoretically active constraints are exactly satisfied. Since in the following examples we investigate only high stiffness due to damping, then, as argued in the end of Section 2.3.4, the projection will not alter the energy bounds provided by Theorem 2.4. The configurations analyzed here are sufficiently regular that the projection procedure can be defined to involve only equality constraints. In the cases where elastic stiffness is present one can use the projection procedure (46) which was not implemented for our examples.

While a contact is clearly added to the active set $\mathscr{A}$ if a collision is detected, we remove a contact from the contact list only when the normal velocity at the contact is positive after solving (17)-(18). In effect, more sophisticated techniques need to be used to avoid potential conflicts between the geometrical constraints and the dynamical resolution [14]. However, for this work we have implemented only the simple active contact removal rule specified above.

Following is a sketch of the algorithm. Aside from the geometrical and dynamical data of the problem, we need to input the intended final time $T$ and timestep $h$.

$v=v^{0}, q=q^{0} ;$ time $=0 ;$

while (time $<T)$

Find $\left(v_{\text {new }}, \tilde{c}_{v}, \tilde{c}_{n}, \tilde{\beta}, \tilde{\lambda}\right)$, a solution of $(17-18)$

if (no collision detected between time and time $+h$ )

else time $=$ time $+h, q=q_{\text {new }}, v=v_{\text {new }}$;

Estimate the collision time time new $_{\text {, collision }}$ position and velocity $q_{\text {new }}$ and $v^{-}$;

Find $\left(v^{+}, \tilde{c}_{v}^{+}, \tilde{c}_{n}^{+}, \tilde{\beta}^{+}, \tilde{\lambda}^{+}\right)$, a solution of (17)-(18) with $q^{(l)}=q_{\text {new }}$ and $h=0$. time $=$ time $_{\text {new }}, v=v^{+}, q=q_{\text {new }}$.

\section{end while}

\section{end if}


Choosing the time step: For the examples described below we observed the exact same behaviour for the linearly implicit algorithm proposed in this work for a large range of time steps. However, we have not implemented a procedure to adapt the time step at this time. Such a procedure can be easily devised between collisions or stick-slip transitions, where the system behaves like a differential algebraic equation and we can, for example, extrapolate to adapt the time step. However, this will not work well if many such collisions or transitions occur in a short period of time, which can happen for several configurations.

In general, one cannot make statements about convergence in a classical sense when high friction is present [3]. Even worse, certain configurations may have multiple solutions which can be quite random in nature (as it happens in a slider-crank with a tight slider with friction [13]). The impossibility of deriving a general rate of convergence theory for high friction prevents the construction of an efficient time step adaptation procedure at this time. The problem of determining appropriate ranges for the time step will be the subject of future research.

\subsection{Solving the linear complementarity problem}

The main effort in simulating one integration step is to solve the mixed LCP (12)-(16). As stated in Theorem 2.2, Lemke's algorithm [5] will find a solution of the LCP in a finite number of steps. If the friction coefficient $\mu \neq 0$, then there is no guarantee that the problem will have a convex solution set. The potential lack of convexity is one of the reasons why other classes of algorithms, such as interior-point algorithms, that perform so well in the case of linear programming, cannot be guaranteed to converge to a solution.

For the results that we report in the following section, we used a Matlab implementation of a variant of Lemke's algorithm [20]. Before using the algorithm, we solved for $v^{(l+1)}$ and $\tilde{c}_{v}$ from Equations (12)-(13) and substituted them in (14)-(16). This resulted in a standard LCP to which the algorithm was applied.

Recently, a hybrid between continuation methods and Lemke's algorithm was proposed. This has resulted in the package PATH, which is specialized in medium scale LCP resolution [21, 22]. Additional information and software is available online at http://www.cs.wisc. edulcpnetl. We used PATH for our simulations and we got the same results as with the algorithm from [20].

\subsection{One-dimensional example}

The first example consists of two bodies on a flat tabletop, with initial distance between centres of 3. The friction coefficient between the bodies and the tabletop is $\mu=0.4$. An external force of $20 \cos (t)$ acts on the body on the left end. All numerical simulations are done with a constant timestep of $0.05 \mathrm{~s}$ for a total of $10 \mathrm{~s}$. Between the two bodies is a damper whose damping parameter will be assigned various values during simulation. The configuration is depicted in Figure 1. For the one-dimensional case we have conducted the following numerical experiments. In all cases we have depicted the positions of the bodies in the $x$ direction with respect to time, as predicted by the numerical method.

(1) For a damping parameter $\gamma=20$, the dynamics was solved both with the Euler LCP from [7] (7) and with the linearly implicit method proposed in this work, (17)-(18). Both bodies have mass 1 . The variation in time of the $x$ positions of the two bodies is presented in Figure 3. 


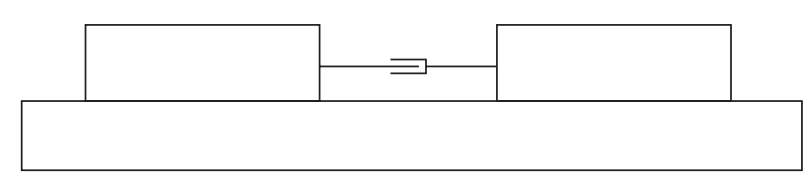

Figure 1. One-dimensional example: two bodies separated by a damper.

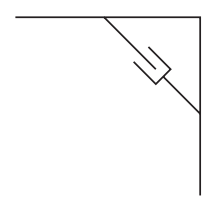

Figure 2. Bidimensional example: two bars articulated at one end with a damper between their centres.

(2) The same experiment was repeated for a damping parameter $\gamma=100$. The results are depicted in Figure 4.

(3) Only the linearly implicit LCP time-stepping scheme (17)-(18) was applied for $\gamma=10^{6}$. Again, both bodies have mass 1. In Figure 5 the results of the simulations are presented together with a graph of the distance between the two bodies.

(4) The linearly implicit LCP time-stepping scheme (17)-(18) was applied for the case where the masses of the bodies were sharply different. In the configuration from Figure 1, the body on the left has mass 1, whereas the body on the right has mass $10^{8}$. Two cases were considered for the damping parameter: $\gamma=20$ and $10^{8}$. The behaviour of the positions of the two bodies is presented in Figure 6.

\subsection{Two-dimensional example}

For a two-dimensional example, we consider two bars of equal length 2 articulated at one end through a revolute joint. Both bars have mass 1 and inertia 1. A graphical configuration of the system is presented in Figure 2. In all cases one of the bars is vertical in the initial position, while the other is placed at an angle of $\pi / 3$ from the first, at which point the system is left to fall toward the tabletop under the influence of gravity. The system falls with 0 initial velocity. There are both a damper and a spring between the centres of the two bars and friction between the bars and the tabletop. In this case we have depicted the successive positions of the bars for various choices of the damping and friction parameters, so as to suggest their predicted trajectory. We have conducted the following experiments, each for a constant time step $h=0.01$, and simulation interval of $10 \mathrm{~s}$.

(1) For the first example, the trajectories are shown in Figure 7 for the articulated two-bar mechanisms for a friction coefficient $\mu=0.15$, elasticity coefficient of $k=100$, and damping coefficients $\gamma$ of 10,1000 , and $10^{8}$. 

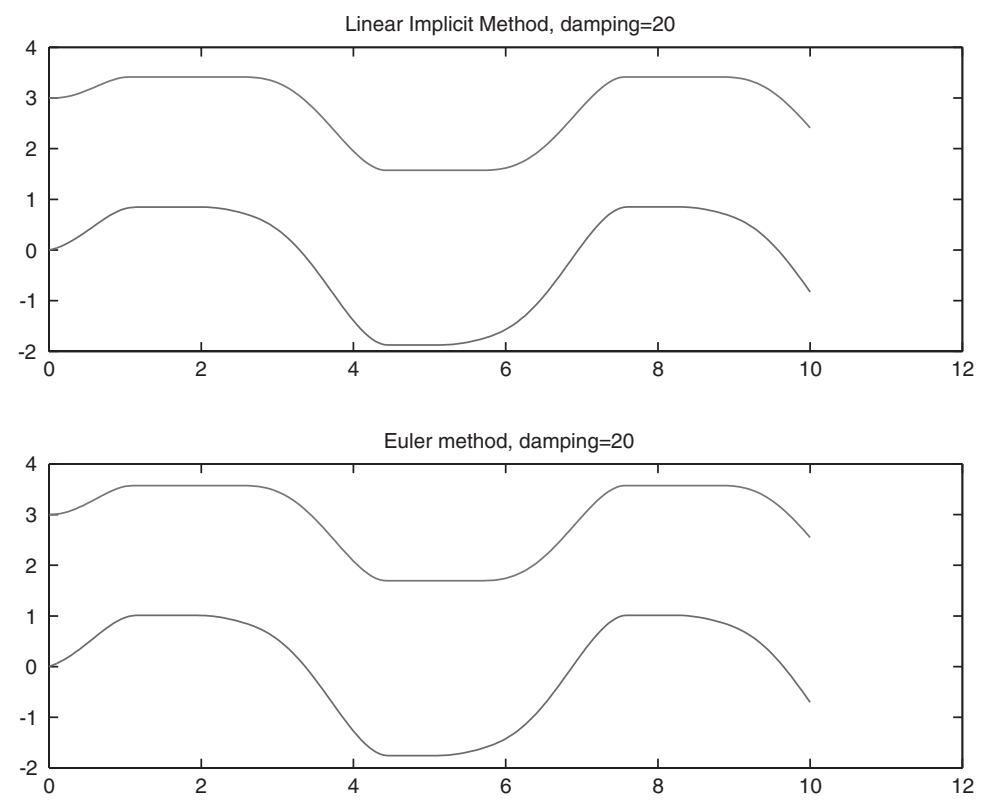

Figure 3. Linearly implicit method versus Euler method for the one dimensional problem with damping coefficient 20. The position of the two bodies (in metres) is plotted as a function of time in seconds.
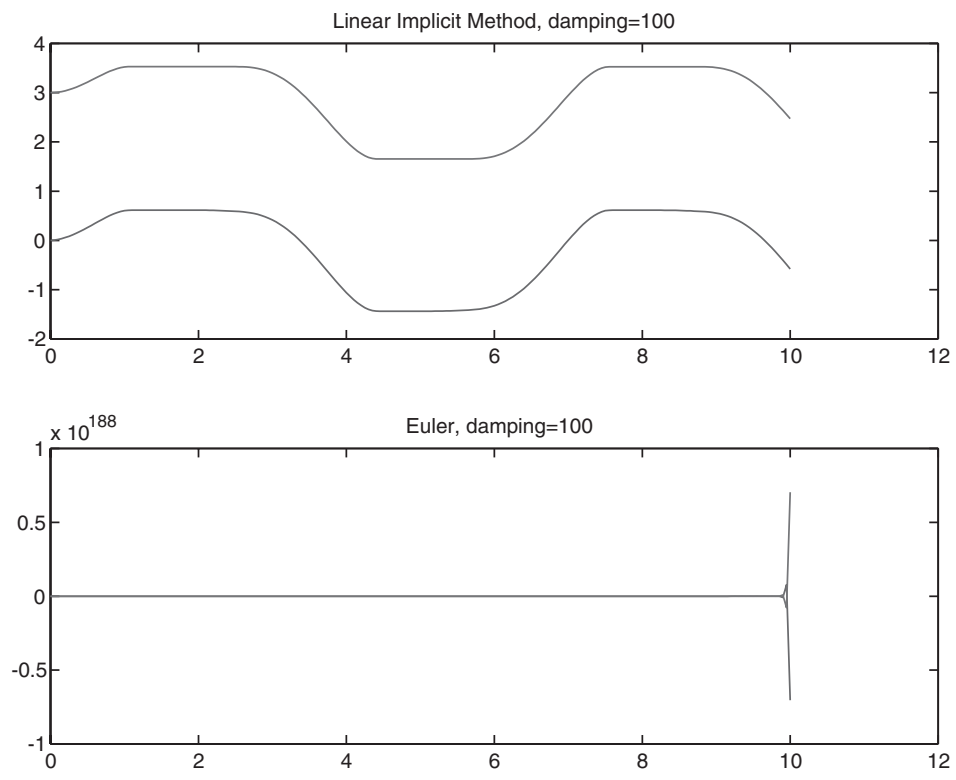

Figure 4. Linearly implicit method versus Euler method for the one-dimensional problem with damping coefficient 100. The position of the two bodies (in metres) is plotted as a function of time in seconds. 

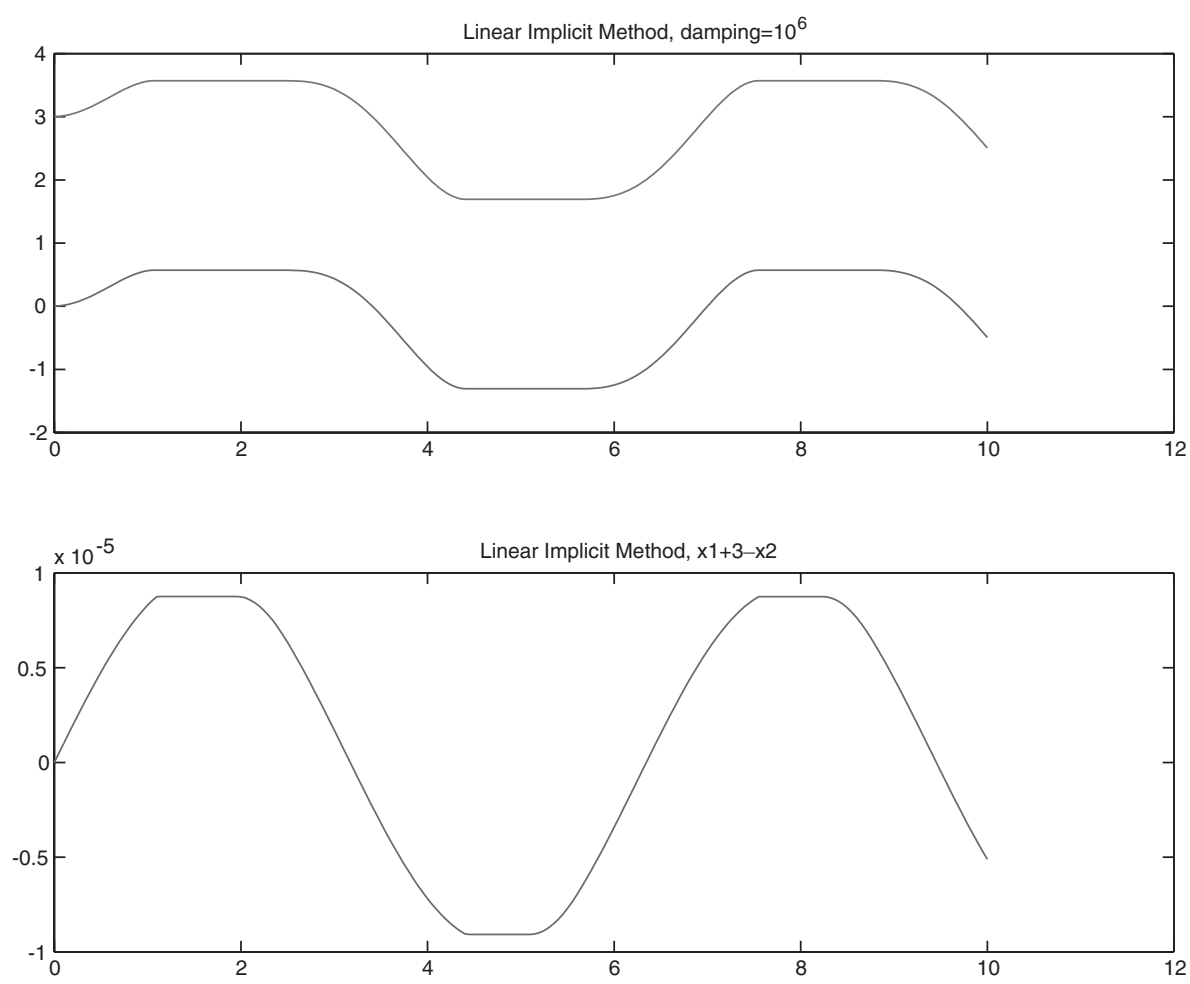

Figure 5. Linear implicit method for damping coefficient $10^{6}$. The position of the two bodies (in metres) is plotted as a function of time in seconds in the first graph. Note the flat regions in the graph that denote the action of static friction. In the second graph, the variation of the distance between the two bodies is plotted as a function of time.

(2) For the second example, whose trajectories are shown in Figure 8, we fix the value of the damping coefficient $\gamma$ at 20, the elasticity coefficient at $k=100$, and we choose the friction coefficient $\mu$ successively to have the values $0.05,0.2$, and 0.5 .

\subsection{Conclusions of the numerical simulations}

The simulations validate the following points:

- For small values of the damping parameter it can be seen from Figure 3 that the output of the linearly implicit scheme (17)-(18) is identical to the explicit one (7). However, even for moderate values of the damping parameter, such as $\gamma=100$, it can be seen from Figure 4 that the explicit scheme (7) results in a catastrophic drift from the solution. We therefore conclude that in order to achieve reasonably large steps, a stabilization technique for stiff systems is necessary even when friction is present.

- The linearly implicit scheme (17)-(18) performs very well even for huge values of the damping parameters, such as $\gamma=10^{6}$ in Figure 5, and $\gamma=10^{8}$ in the last plot of Figure 7, as predicted by Theorem 2.6 and Corollary 2.5. 

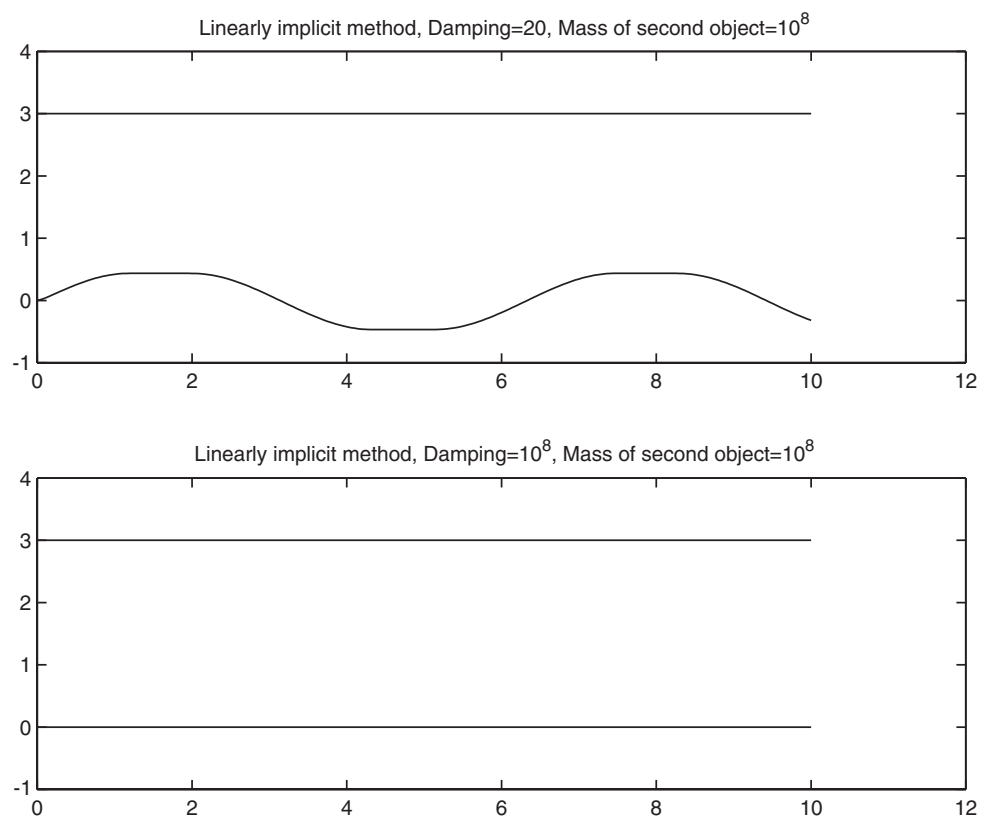

Figure 6. Behaviour of linearly implicit method for large differences in masses between objects $\left(m_{1}=1, m_{2}=10^{8}\right)$. The position of the two bodies (in metres) is plotted as a function of time in seconds in the first graph. The flat regions in the first graph again denote the effect of the static friction.

- From Figures 3-7, we observe that the behaviour of a stiff system as predicted by the linearly implicit scheme (17)-(18) approaches the behaviour of the system when the damper and spring are replaced with a rigid joint. This is again consistent with the result of Theorem 2.6, since in the case where the stiffness does not originate in spring forces, the initial position of the spring is not relevant for the theorem to apply.

- We also demonstrate the effects of friction. For the one-dimensional case the effect of static friction is immediately seen in Figure 5, for the duration where the graph of the $x$ coordinates of the two bodies is flat, indicating that the bodies do not move. Figure 8 shows that increasing the friction coefficient has the effect of stopping the two-bar mechanism earlier.

- We demonstrate that our method is stable in the presence of configurations with sharply different masses, as seen in Figure 6. In the case with low damping, the motion of the second body is imperceptible, due to its large mass, whereas the first body is allowed to move due to the small damping $\gamma=20$. In the high damping case, $\gamma=10^{8}$, the large damping parameter forces the damper to act as a rigid link, and the large mass of the second body thus forces both bodies to stay essentially unmoved. In Figure 6 we see that the numerical scheme simulates exactly this type of behaviour. This validates our findings at the end of Section 2.3.5, that the numerical scheme is stable with respect to large masses. 

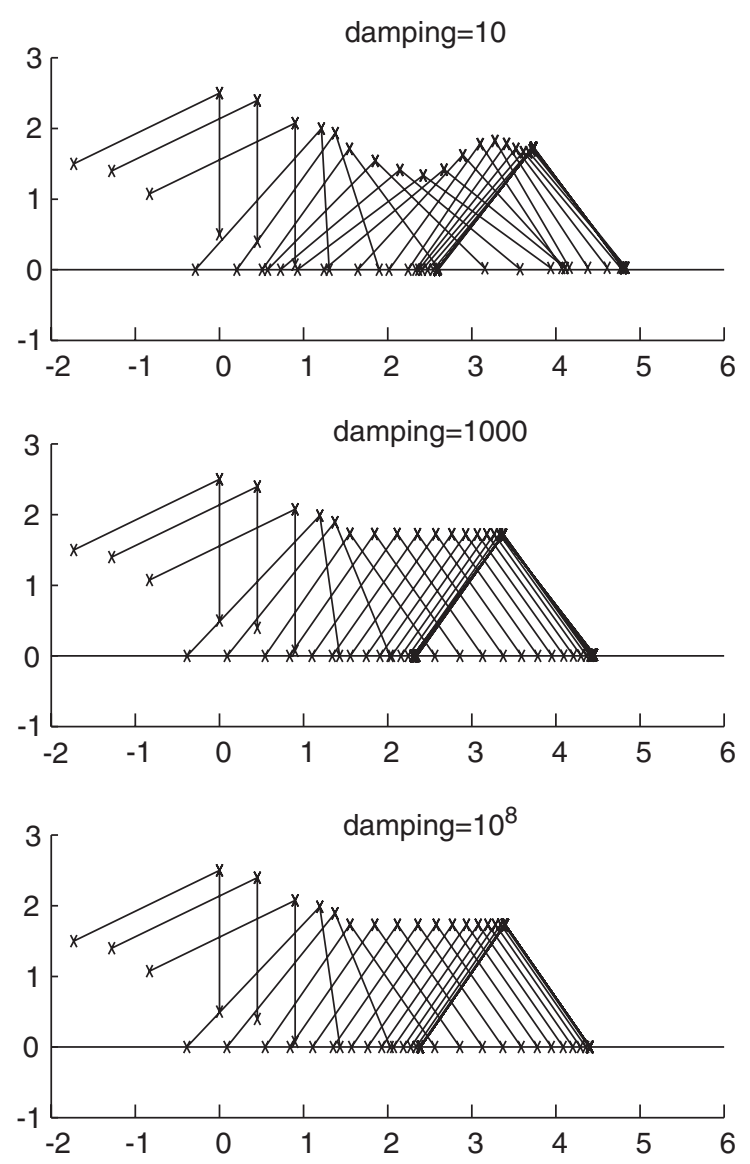

Figure 7. Simulations for the link with a damper between the centres of mass after varying the damping coefficients.

- We have observed an identically stable behaviour when increasing the elasticity parameter, although we do not report the results here. This behaviour is consistent with our observation concerning total energy from Lemma 2.4 , though it is only partially explained by our observation. The theoretical analysis of this behaviour will be the subject of future research.

\section{CONCLUSIONS}

We present a time-stepping method for rigid multibody dynamics with contact, friction and stiff external forces. We show that the method is well defined for sufficiently small time step and is unconditionally consistent for the case where the stiff force originates in springs and dampers attached between two points of the system. We also show that if the damping 

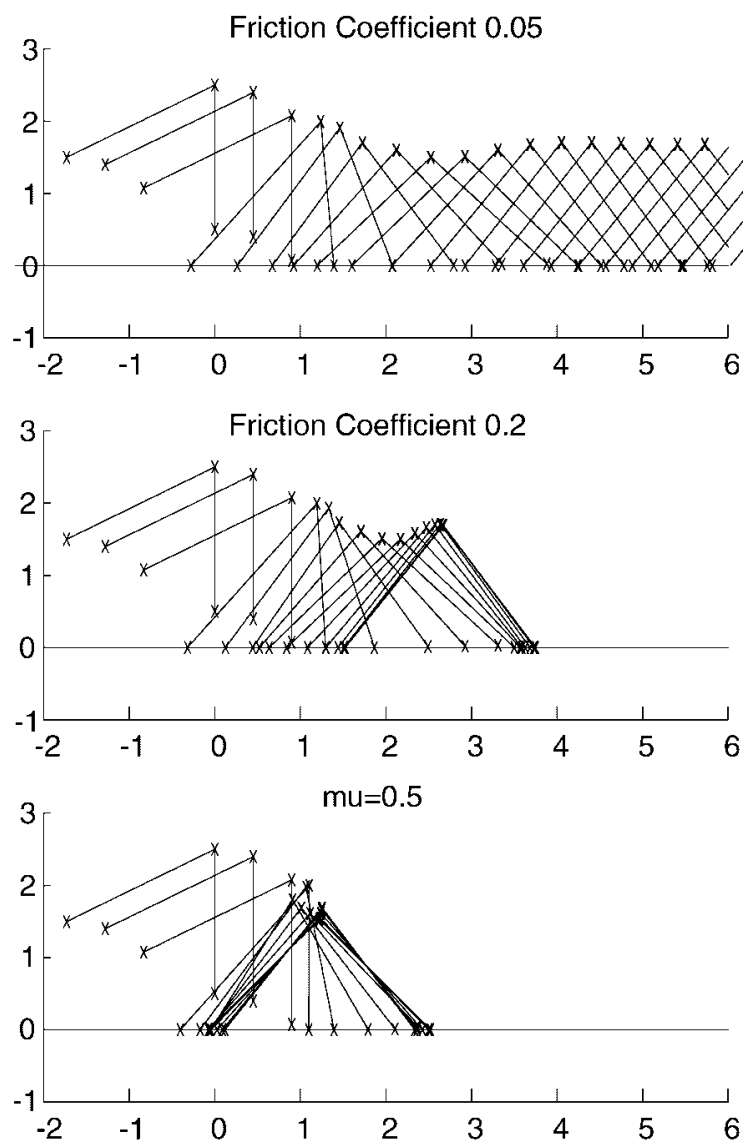

Figure 8. Simulations for the link with a damper between the centres of mass for varying the friction coefficients.

coefficients go to infinity, then the total energy stays bounded, and the behaviour of the system approaches the behaviour of a system with the dampers replaced by rigid links.

Our work extends previous consistency results for time-stepping velocity-impulse schemes $[3,7]$ for the case of stiff external forces, while still requiring the resolution of linear complementarity subproblems that can be solved with Lemke's algorithm, for example.

It is also clear that the new scheme (17)-(18) is asymptotically equivalent to the original explicit scheme (7) for small $h$. Hence the convergence results as $h \rightarrow 0$ apply to show that the numerical solution approaches the solution of a measure differential inclusion under reasonable assumptions.

\section{ACKNOWLEDGEMENTS}

Thanks to Michael Ferris for providing a Matlab version of the algorithm in Reference [20]. Thanks to Todd Munson for advice and support concerning the use of PATH. Thanks to the anonymous referee 
and Chris Hecker who suggested discussing the case involving stiffness due to large mass parameters. Thanks to the anonymous referees for the suggestions that have resulted in an improved paper. This work was supported by the Mathematical, Information, and Computational Sciences Division subprogram of the Office of Advanced Scientific Computing, U.S. Department of Energy, under Contract W-31-109Eng-38. This work was also supported by award DMS-9973071 of the National Science Foundation. The work of F. A. Potra has been supported in part by the National Science Foundation, grant DMS9996154.

\section{REFERENCES}

1. Murray RM, Li Z, Sastry SS. Robotic Manipulation. CRC Press: Boca Raton, FL, 1993.

2. Baraff D. Issues in computing contact forces for non-penetrating rigid bodies. Algorithmica 1993; 10:292-352.

3. Stewart D. Rigid-body dynamics with friction and impact. SIAM Review 2000; 42(1):3-29.

4. Haug EJ, Wu SC, Yang SM. Dynamic mechanical systems with Coulomb friction, stiction, impact and constraint addition-deletion I: Theory. Mechanism and Machine Theory 1986; 21(5):407-416.

5. Cottle RW, Pang J-S, Stone RE. The Linear Complementarity Problem. Academic Press: Boston, 1992.

6. Lötstedt P. Mechanical systems of rigid bodies subject to unilateral constraints. SIAM Journal on Applied Mathematics 1982; 42(2):281-296.

7. Anitescu M, Potra FA. Formulating rigid multi-body-dynamics with contact and friction as solvable linear complementarity problems. Nonlinear Dynamics 1997; 14:231-247.

8. Stewart DE, Trinkle JC. An implicit time-stepping scheme for rigid-body dynamics with inelastic collisions and Coulomb friction. International Journal for Numerical Methods in Engineering 1996; 39:2673-2691.

9. Featherstone R. Robot Dynamics Algorithms. Kluwer Academic Publishers: Boston, 1987.

10. Trinkle JC, Pang JS, Sudarsky S, Lo, G. On dynamic multi-rigid-body contact problems with Coulomb friction. Zeitschrift fur Angewandte Mathematik und Mechanik 1997; 77:267-279.

11. Trinkle D. Stabilized fixtures, presented at the First Computational Science and Engineering Conference, Washington, DC, September 2000.

12. Haug EJ. Computer Aided Kinematics and Dynamics of Mechanical Systems. Allyn and Bacon: Boston, 1989.

13. Anitescu M, Cremer J, Potra FA. Formulating 3D contact dynamics problems. Mechanics of Structures and Machines 1996; 24(4):405-437.

14. Cremer J, Vanecek G. Building simulations for virtual environments. Proceedings of the IFIP International Workshop on Virtual Environments, Coimbra, Portugal, October 1994.

15. Hairer E, Wanner G. Solving ordinary differential equations II-stiff and differential-algebraic problems. Springer Series in Computer Mathematics, vol. 14. Springer: Berlin, 1996.

16. Anitescu M, Cremer J, Potra FA. On the existence of solutions to complementarity formulations of contact problems with friction. In Complementarity and Variational Problems: State-of-the-Art. Proceedings of the International Conference on Complementarity Problems, 1-4 November 1995, Baltimore. SIAM: Philadelphia, 1996; 12-21.

17. Anitescu M, Stewart D, Potra FA. Time-stepping for three-dimensional rigid body dynamics. Computer Methods in Applied Mechanics and Engineering 1999; 177(3-4):183-197.

18. Stewart D. Convergence of a time-stepping scheme for rigid body dynamics and resolution of Painleve's problems. Archive for Rational Mechanics and Analysis 1998; 145(3):215-260.

19. Bonnans JF, Shapiro A. Perturbation Analysis of Optimization Problems. Springer: New York, 2000.

20. Cao M, Ferris MC. A pivotal method for affine variational inequalities. Mathematics of Operations Research 1996; 21:44-64.

21. Dirkse SP, Ferris MC. The PATH solver: a non-monotone stabilization scheme for mixed complementarity problems. Optimization Methods and Software 1995; 5:123-156.

22. Munson TS. Algorithms and environments for complementarity. Ph.D Thesis, Department of Computer Science, University of Wisconsin-Madison, 2000.

23. Anitescu M. Degenerate nonlinear programming with a quadratic growth condition. SIAM Journal on Optimization 2000; 10(4):1116-1135. 